\title{
High Order Modes in Project-X Linac
}

A. Sukhanov*, A. Lunin, V. Yakovlev, M. Awida, M. Champion, C. Ginsburg, I. Gonin, C. Grimm, T. Khabiboulline, T. Nicol, Yu. Orlov, A. Saini, D. Sergatskov, N. Solyak, A. Vostrikov

Fermilab, Batavia, IL 60510, USA

\begin{abstract}
Project-X, a multi-MW proton source is now under development at Fermilab. In this paper we present study of high order modes (HOM) excited in continues-wave (CW) superconducting linac of Project-X. We investigate effects of cryogenic losses caused by HOMs and influence of HOMs on beam dynamics. We find that these effects are small. We conclude that HOM couplers/dampers are not needed in the Project-X SC RF cavities.
\end{abstract}

Keywords: superconducting proton linac, high order modes, beam dynamics

\section{Introduction}

Fermilab is currently developing a multi-MW proton source, Project-X (PX) [1], which will provide intense muon, kaon, neutrino and nuclei beams for broad and diverse program at the intensity frontier of particle physics. PX will allow study of applications of proton accelerators for energy production and nuclear waste transmutation in accelerator-driven subcritical systems. And, eventually, Project-X may become an integral part and driver for the future Fermilab Neutrino Factory and/or Muon Collider. Figure 1 shows a general layout of Project-X.

A key component of Project-X is the CW SRF linac, which accelerates bunches of $\sim 16 \cdot 10^{7} \mathrm{H}^{-}$ions from $2.1 \mathrm{MeV}$ to $3 \mathrm{GeV}$. The linac layout

*ais@fnal.gov

${ }^{1}$ Operated by Fermi Research Alliance, LLC under Contract No. De-AC02-07CH11359 with the United States Department of Energy. 


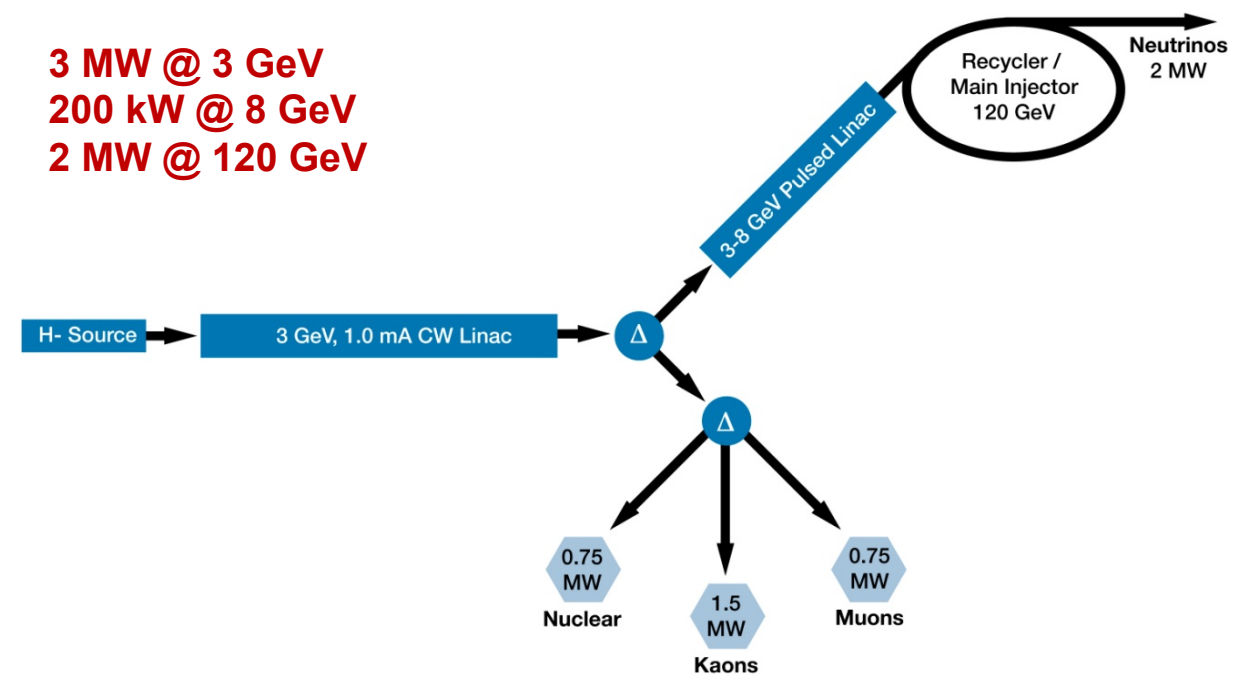

Figure 1: Project-X layout.

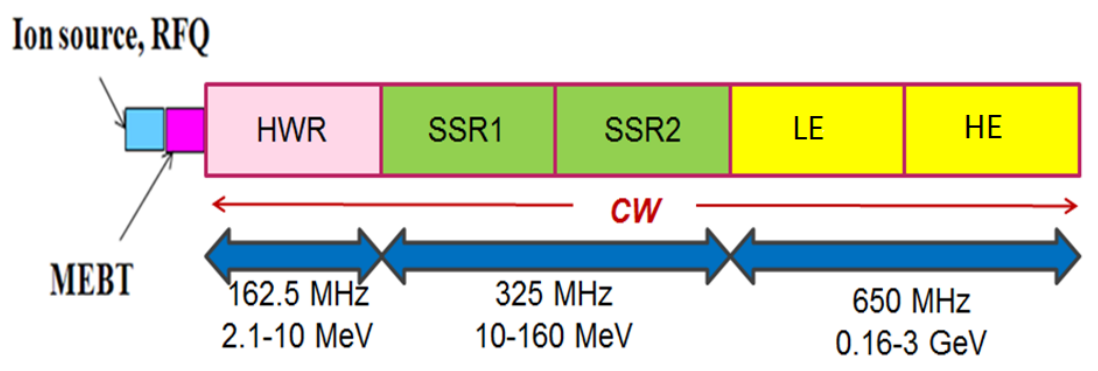

Figure 2: Layout of Project-X CW linac.

is shown in Figure 2. It includes a section of half-wave resonators (HWR, Figure 3a) at $162.5 \mathrm{MHz}$, two sections of single-spoke cavities (SSR1, Figure 3b, and SSR2, Figure 3c) at $325 \mathrm{MHz}$, and low energy (LE) and high energy (HE) sections of 5-cell elliptical cavities at $650 \mathrm{MHz}$ (see Figure 4). Two designs exist for the LE section, one from JLab [3] and one from Fermilab [4]. The Fermilab design has been used for the calculations in this paper. The conclusions of this paper are not expected to vary significantly between two design. Table 1 lists nominal beam velocity $\beta_{G}$, energy range, total number of cavities, number of focusing magnets and number of cryomodules (CM), length of cryomodules, and proposed packaging of cavities 
and magnets inside cryomodules.

Table 1: Cavities and cryomodules in Project X linac.

\begin{tabular}{|l|c|c|c|c|c|}
\hline Section & $\beta_{G}$ & $\begin{array}{c}\text { Freq. } \\
\mathrm{MHz}\end{array}$ & $\begin{array}{c}\text { Energy } \\
\mathrm{MeV}\end{array}$ & $\begin{array}{c}\text { Cav/Mag/ } \\
\text { CM }\end{array}$ & $\begin{array}{c}\text { Type of cavities, } \\
\text { magnets, CM length }\end{array}$ \\
\hline HWR & 0.11 & 162.5 & $2.1-10$ & $8 / 8 / 1$ & HWR, solenoid, $5.26 \mathrm{~m}$ \\
SSR1 & 0.22 & 325 & $10-32$ & $16 / 8 / 2$ & SSR, solenoid, $4.76 \mathrm{~m}$ \\
SSR2 & 0.47 & 325 & $32-160$ & $36 / 30 / 4$ & SSR, solenoid, 7.77 m \\
LE 650 & 0.61 & 650 & $160-520$ & $42 / 14 / 7$ & 5-cell ellip., doublet, 7.1 m \\
HE 650 & 0.9 & 650 & $520-3000$ & $152 / 19 / 19$ & 5-cell ellip., doublet, $11.2 \mathrm{~m}$ \\
\hline
\end{tabular}

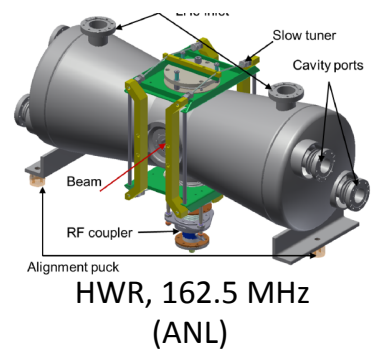

a)

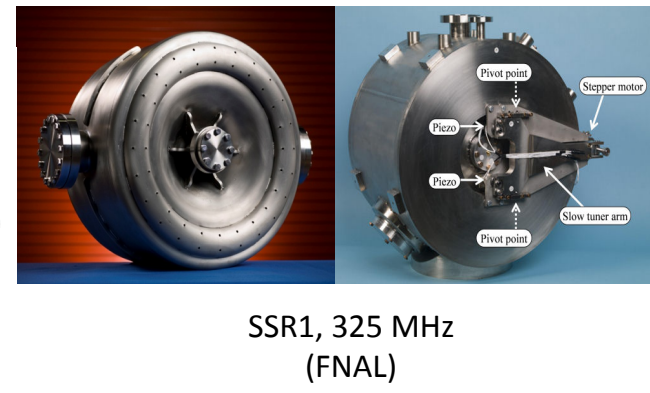

b)

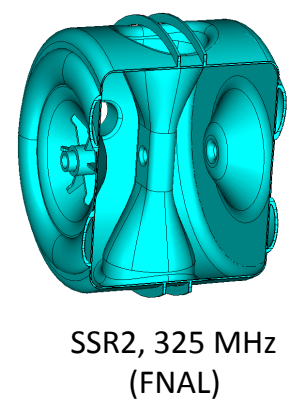

c)

Figure 3: a) Half-wave, b) single spoke 1 (bare and dressed), and c) single spoke 2 cavities of Project-X.

RF design parameters of half-wave and single-spoke resonators are listed in Table 2. Table 3 presents RF design parameters of the both LE and HE $650 \mathrm{MHz}$ cavities. In these tables parameter $G$ is cavity geometry constant (see, for example, $[2]$, p. 45, eq. 2.52). Parameters $E_{a c c},(R / Q),{ }^{2}$ that depend on beam velocity $\beta$, are shown for the optimal velocity, $\beta_{\text {opt }}$. Intrinsic quality factor $Q_{0}$ and heat load are given at $2 \mathrm{~K}$.

\subsection{Importance of $H O M$ investigation}

Excitation of high order modes in SRF cavities is always a concern. Heating caused by beam power lost to HOMs adds to the cryogenic losses and

\footnotetext{
${ }^{2}$ Note, that in this paper we use accelerator definition of $(R / Q)$ value, as in [2], p. 47 .
} 
Table 2: Half-wave and single-spoke resonator cavities.

\begin{tabular}{|c|c|c|c|c|c|c|c|}
\hline Type & $\begin{array}{c}\text { Beam pipe } \\
\varnothing, \mathrm{mm}\end{array}$ & $\begin{array}{c}\max V_{a c c} \\
\mathrm{MeV}\end{array}$ & $\begin{array}{c}\max E_{a c c} \\
\mathrm{MV} / \mathrm{m}\end{array}$ & $\begin{array}{c}B_{\max } \\
\mathrm{mT}\end{array}$ & $\begin{array}{c}(R / Q) \\
\Omega\end{array}$ & $\begin{array}{c}G \\
\Omega\end{array}$ & $\begin{array}{c}Q_{0} \\
\times 10^{10}\end{array}$ \\
\hline HWR & 33 & 1.8 & 40 & 62 & 225 & 47.7 & 1.0 \\
SSR1 & 30 & 1.95 & 28 & 70 & 242 & 84 & 1.1 \\
SSR2 & 40 & 3.34 & 32 & 60 & 292 & 109 & 1.3 \\
\hline
\end{tabular}

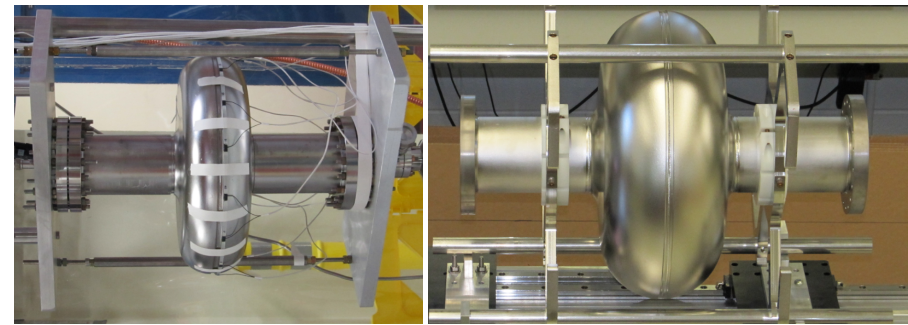

Single-cell models: LE $650 \mathrm{MHz}$ (JLAB)

a)
HE $650 \mathrm{MHz}$ (FNAL)

b)

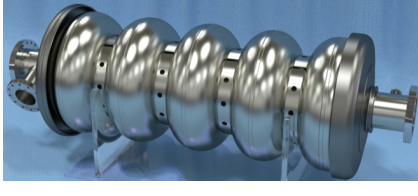

5-cell model, HE650

c)

Figure 4: Elliptical cavities in Project-X linac: a) LE $650 \mathrm{MHz}$ single-cell prototype cavity (JLab design); b) HE $650 \mathrm{MHz}$ single-cell prototype cavity (Fermilab design); and c) HE $650 \mathrm{MHz} 5$-cell prototype cavity.

increases operational cost of the linac. Interaction of the beam with excited HOMs deteriorates the quality of the beam.

One of the ways to reduce HOMs is to equip accelerating cavities with couplers, which re-direct and dump HOMs outside of the cavities. The drawback of this solution is that the HOM couplers are vulnerable, expensive, and complicated part of the SC accelerating structure and not effective in the full range of the HOM frequencies. HOM couplers add extra hardware to cryomodules: cables, feed-through, connectors, loads, etc. They are susceptible to multipactoring and damage of feed-throughs.

Experience with super-conducting proton linac at SNS shows that HOM couplers may limit cavity performance and reduce operation reliability. Observed power loss to HOMs at SNS is very small and HOM couplers were found to be not necessary [5].

Power loss to HOMs and, respectively, their adverse effect on beam quality and heat load depend on the beam current, beam spectrum, and the 
Table 3: $650 \mathrm{MHz}$ elliptical cavities.

\begin{tabular}{|l|c|c|c|}
\hline Parameter & Units & LE 650 & HE 650 \\
\hline$\beta_{G}$ & & 0.61 & 0.9 \\
$L_{\text {cav }}$ & $\mathrm{mm}$ & 703 & 1038 \\
$(R / Q)$ & $\Omega$ & 378 & 638 \\
$G$ & $\Omega$ & 191 & 255 \\
$\max V_{\text {acc }}$ & $\mathrm{MeV}$ & 11.7 & 17.7 \\
$E_{\text {acc }}$ & $\mathrm{MV} / \mathrm{m}$ & 16.6 & 17 \\
$\max E_{\text {surf }}$ & $\mathrm{MV} / \mathrm{m}$ & 37.5 & 34 \\
$\max B_{\text {surf }}$ & $\mathrm{mT}$ & 70 & 61.5 \\
$Q_{0}$ & $\times 10^{10}$ & 1.5 & 2.0 \\
Maximum heat load & $\mathrm{W}$ & 24 & 24 \\
\hline
\end{tabular}

Table 4: Incoherent HOM power loss in Project-X, SNS and ILC.

\begin{tabular}{|l|c|c|c|}
\hline & Project-X & SNS & ILC \\
\hline$I_{\mathrm{av}}, \mathrm{mA}$ & 1 & 1 & 0.048 \\
$Q_{b}, \mathrm{pC}$ & 25.6 & 58 & 3200 \\
$k_{\text {loss }}, \mathrm{V} / \mathrm{pC}$ & 2 & 1.1 & 13.4 \\
$P_{\mathrm{av}}, \mathrm{mW} /$ cavity & 51 & 64 & 2065 \\
\hline
\end{tabular}

cavity HOM spectrum. Assuming that each beam bunch excites HOMs in cavities independently from the other bunches ("incoherent losses") an average HOM power per cavity can be estimated as following: $P_{a v}=k_{l o s s} q_{b} I_{a v}$, where $k_{\text {loss }}$ is a loss-factor, $q_{b}$ is a bunch charge, and $I_{a v}$ is an average beam current. Table 4 compares incoherent losses in Project-X, SNS, and ILC superconducting RF structures. One can see that in the ILC linac the HOM power loss is very high hence HOM dampers are necessary. All the $1.3 \mathrm{GHz}$ ILC cavities are equipped with HOM couplers, that reduce loaded quality factors of longitudinal and transverse HOMs to $10^{5}$ and work successfully at DESY. However, some problems with HOM couplers were encountered during long pulse operation [6]. In the SNS cavities no measurable HOM signals from beam were observed. Also, analysis of collective beam effects in SNS, such as a beam break-up and a klystron-type instability, do not show critical influence of high order modes on the beam dynamics [5]. 


\subsection{Scope of investigation}

The goal of this paper is to understand the HOM effects on the heat load and the beam dynamics in the Project-X superconducting RF cavities, and to decide whether HOM dampers are needed in the high and low energy sections of the CW linac. We focus our investigation on the following topics: calculation of loss factors (Section 2); investigation of beam spectrum for different modes of operation of Project-X (Section 3); simulation and measurement of cavity spectrum for all sections of linac (Section 4); estimation and measurement of spread of HOM frequencies and impedances; estimation of cryogenic loss taking into account non-propagating and propagating modes; estimation of the longitudinal and transverse beam emittance dilution caused by HOMs (Section 5); investigation of stability of HOMs with respect to tuning and detuning of the accelerating mode (Section 6); estimation of longitudinal and transverse beam instabilities (Section 7); comparison to the existing proton superconducting linacs (SNS); study of effects of microphonics for different HOMs (Section 8.1).

Project-X has complicated beam timing structure. Beam current is rather small and beam is non-relativistic. Beam passes linac only once, so there is no feedback as in circular accelerators. All the above factors affect excitation of high order modes.

\section{Incoherent losses}

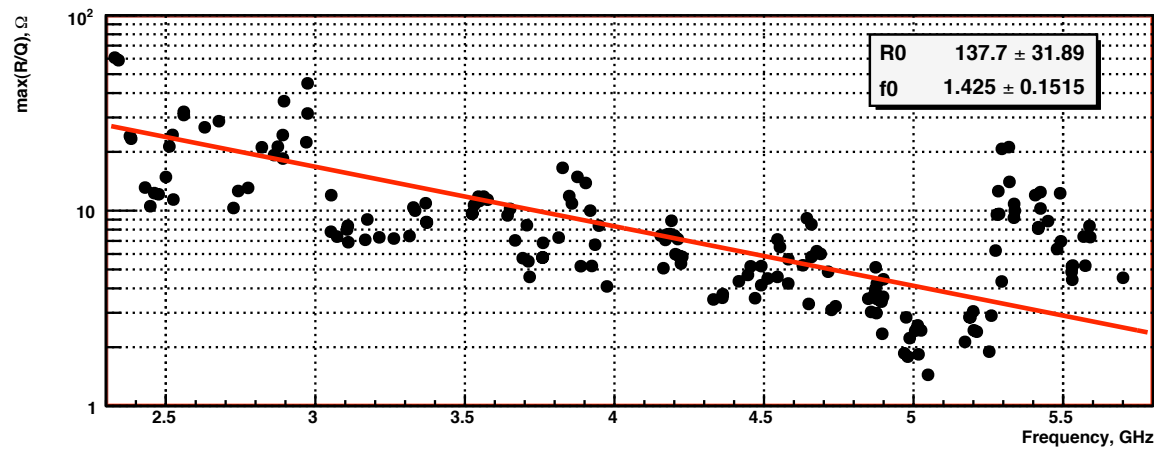

Figure 5: Impedance of longitudinal HOMs in $\mathrm{HE} 650 \mathrm{MHz}$ section of Project-X calculated with SuperLANS. Data are approximated by an exponential function $(R / Q)=$ $R_{0} \exp \left(-f / f_{0}\right)$, with $R_{0}=137.7 \Omega$, and $f_{0}=1.425 \mathrm{GHz}$. 
We use SuperLANS [7] program $^{3}$ to calculate spectrum of HOMs in HE $650 \mathrm{MHz}$ sections of Project-X. The cavity model and geometry are described in more details in Section 4.1. In general, modes with the frequencies above the beam pipe cut-off frequency ${ }^{4}$ escape, or propagate from the cavity into beam pipe and can be removed from the accelerator by HOM couplers and absorbers. But when multiple cavities are put together in a linac at the regular intervals, conditions may arise where some of these modes are effectively trapped inside the cavity chain. Without experimental data from real linac or a complete model of entire accelerator it is not possible to predict which of the propagating modes may be trapped. We use a conservative approach and assume that all propagating modes are trapped. Impedance of propagating modes depends on the length of the beam pipe between adjacent cavities. In our calculations we model single cavity with beam pipes attached at the cavity ends. The beam pipes are closed with perfect electric conductor (PEC) flanges. We vary length of the beam pipes in the range from 10 to $50 \mathrm{~cm}$ in increments of $1 \mathrm{~mm}$ and calculate cavity spectrum for each length. We then use the maximum value of impedance of the propagating modes with respect to the beam pipe length. Figure 5 shows frequency dependence of the impedance of the longitudinal HOMs. The impedance quickly drops with the frequency and can be approximated by an exponential function: $(R / Q)=R_{0} \exp \left(-f / f_{0}\right)$ with $R_{0}=137.7 \Omega$ and $f_{0}=1.425 \mathrm{GHz}, f$ is the $\mathrm{HOM}$ frequency.

The effect of beam velocity on HOMs can be demonstrated using the following consideration (see Figure 6). Frequencies (and number) of HOMs excited by a beam bunch, passing trough an SRF structure, depend on the characteristic size of the EM field distribution on the wall of the beam pipe at the cavity entrance, $\sigma_{\text {field. }}$. In linacs with relativistic beam, such as ILC [8], XFEL [9] or NGLS [10], the field distribution is essentially disk-like and $\sigma_{\text {field }} \sim \sigma_{\text {bunch }}$. Bunch EM field spectrum has frequencies up to $f_{\max } \sim$

\footnotetext{
${ }^{3}$ SuperLANS is fast and simple program which allows for quick finding of monopole, dipole and quadrupole eigen-modes in axially symmetric RF structures.

${ }^{4}$ The beam pipe cut-off frequency the lowest frequency of the beam pipe waveguide modes. The lowest propagating TM mode of the cylindrical beam pipe of radius $a$ has the cut-off frequency $f_{\text {cut-off }}=\frac{c t_{01}}{2 \pi a}$, where $c$ is speed of light and $t_{01} \approx 2.4048$ is the $1^{\text {st }}$ zero of Bessel function of $0^{t h}$ order: $J_{0}\left(t_{01}\right)=0$. For example, HE $650 \mathrm{MHz}$ section of Project$\mathrm{X}$ linac has the beam pipe of radius $a=5 \mathrm{~cm}$ and the cut-off frequency $f_{\text {cut-off }} \approx 2.3$ GHz.
} 

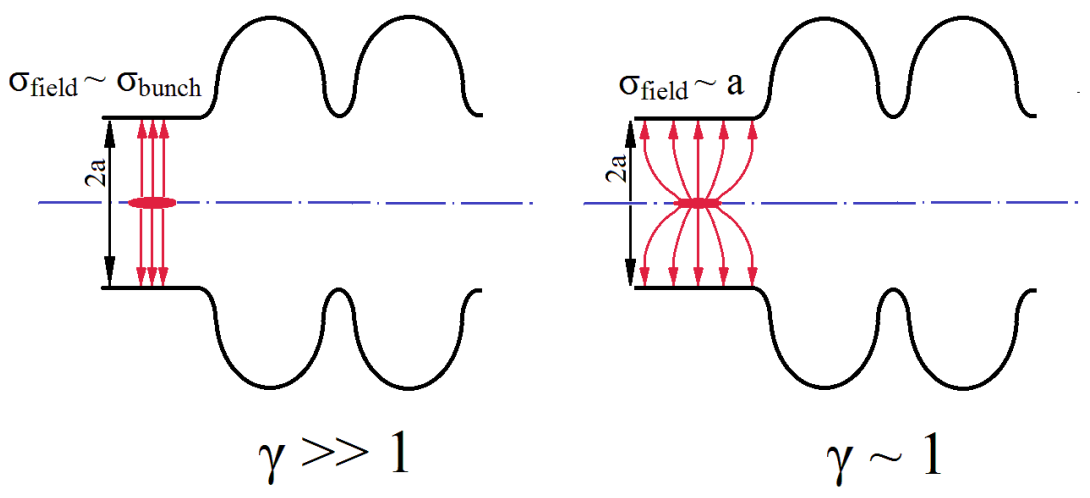

Figure 6: Effect of beam velocity on HOM excitation.

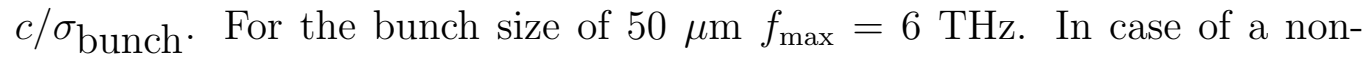
relativistic beam, field lines diverge much more, and the characteristic size of field distribution is of the order of the beam pipe radius, $\sigma_{\text {field }} \sim a$ and $f_{\max } \sim c / a$. If the beam pipe radius is $50 \mathrm{~mm}$, only frequencies below 6 $\mathrm{GHz}$ are present in the bunch field spectrum. Taking into account rapid exponential decay of HOM spectrum as shown in Figure 5, we conclude that effectively only HOMs below few $\mathrm{GHz}$ will be be excited by the nonrelativistic beam in the Project-X linac.

We perform simulation of incoherent losses using time-domain calculations [11] in CST Studio program [12]. Results are shown in Figure 7. Losses are well below $100 \mathrm{~mW} /$ cavity in $\mathrm{HE} 650 \mathrm{MHz}$ section, and of the order of magnitude smaller in LE $650 \mathrm{MHz}$ part.

\section{Beam spectrum}

Amplitude and frequency of the main lines of the beam current spectrum directly affect probability of excitation and strength of high order modes in superconducting accelerating RF structures.

The bunch sequence frequency in Project-X is $162.5 \mathrm{MHz}$. A broad-band chopper provides the beam structure needed for the experiments. Three regimes of operation are considered:

1. A bunch timing structure required for muon, kaon, and nuclear experiments at $3 \mathrm{GeV}$. Figure 8 shows one full period (approximately $1 \mu \mathrm{s}$ ) of the bunch train in this regime. Average beam current is $1 \mathrm{~mA}$. The de- 


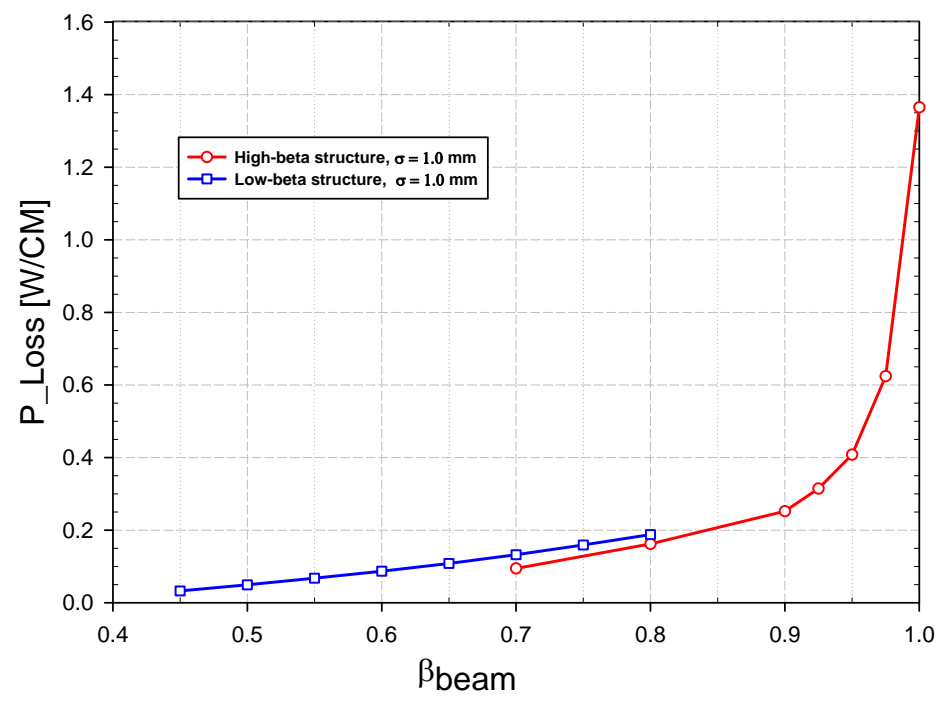

Figure 7: Incoherent losses per cryomodule in LE and HE $650 \mathrm{MHz}$ sections of Project-X linac.

livered power is 700/1540/770 kW for muon/kaon/nuclear experiments, respectively.

2. A $3 \mathrm{GeV}$ structure combined with $10 \mathrm{~Hz} 5 \%$ duty factor (DF) pulses for injection into the Fermilab Main Injector (MI) for the $120 \mathrm{GeV}$ neutrino program; $33 \%$ of the bunches within pulses are removed to match the phase of $\sim 50 \mathrm{MHz}$ of $\mathrm{MI} \mathrm{RF}$.

3. A $3 \mathrm{GeV}$ structure combined with $15 \mathrm{~Hz} 10 \%$ DF pulses, which can be used to drive a future Muon Collider; within these pulses, bunches are structured in $1 \mu \mathrm{s}$ "micro"-pulses with a $50 \%$ DF. The mean value of pulsed current in this mode is $5 \mathrm{~mA}$.

The spectrum for an idealized $3 \mathrm{GeV}$ beam structure, assuming very short bunches of equal charge and the absence of timing jitter, is shown in Figure 9. The main lines are harmonics of $162.5 \mathrm{MHz}, 20 \mathrm{MHz}$ and 10 $\mathrm{MHz}$ corresponding to the three components of the beam for muon, kaon and nuclei experiments. The sideband lines of $1 \mathrm{MHz}$ quickly fall below $0.1 \mathrm{~mA}$.

Because of the small duty factor of $5 \%$ and chopping off $33 \%$ of bunches during the MI pulses in the mode 2 , there are only very minor modifications 


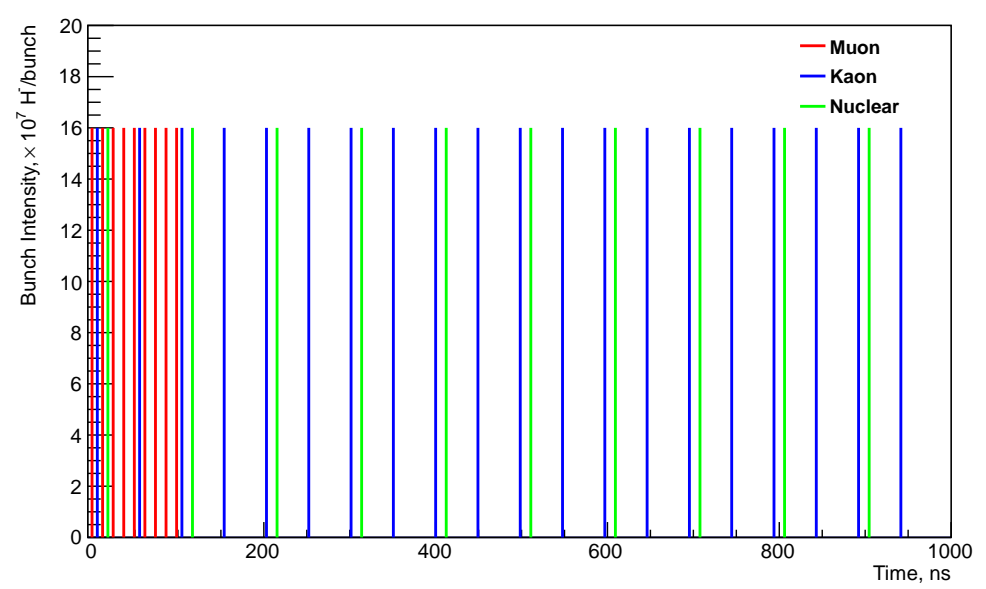

Figure 8: Beam structure for $3 \mathrm{GeV}$ program.

to the beam current spectrum of the $3 \mathrm{GeV}$ timing structure. Indeed, in this case, $10 \mathrm{~Hz}$ sidebands are added to $1 \mathrm{MHz}$ lines of the $3 \mathrm{GeV}$ spectrum shown in Figure 9. The amplitude of these lines falls quickly from $0.1 \mathrm{~mA}$ to the sub- $\mu \mathrm{A}$ level. Since we are already considering variations of HOM frequencies of the order of $1 \mathrm{MHz}$, the effect of MI pulses on the power loss is negligible.

The spectrum of the beam current corresponding to the pulses for Muon Collider operation is shown in Figure 10. The main lines of this spectrum are the harmonics of the bunch sequence frequency, $162.5 \mathrm{MHz}$, with an amplitude of about $1 \mathrm{~mA}^{5}$. The amplitude of the sideband lines, separated by $2 \mathrm{MHz}$ (due to $50 \%$ DF during the "micro"-pulse), drops quickly below $0.1 \mathrm{~mA}$.

\section{Cavity spectrum}

In this section we evaluate spectra and impedances of the high order modes in the Project-X cavities. The main contribution into HOM losses comes from the modes with the highest impedance and the ones with the frequencies in proximity of the main lines of beam current spectrum.

\footnotetext{
${ }^{5}$ Note that the average value of pulsed current is $5 \mathrm{~mA}$, but there is a $10 \%$ pulse $\mathrm{DF}$ and $50 \%$ DF at the $1 \mu \mathrm{s}$ "micro"-pulse level.
} 


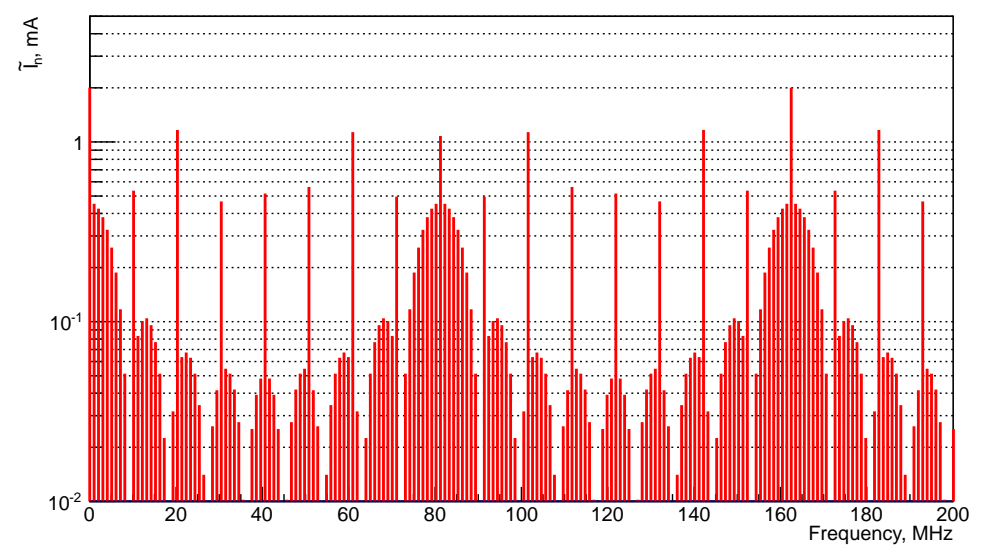

Figure 9: Beam spectrum of $3 \mathrm{GeV}$ program.

\section{1. $650 \mathrm{MHz}$ cavities}

Figure 11 shows a parameterization of geometry of the half-cell of an elliptical cavity. There is rotational symmetry with respect to the cavity axis (side $L$ on Figure 11). The full accelerating cavity is made of few cells that may have different geometry in order to achieve better field flatness on the cavity axis. Parameters of half-cells of $650 \mathrm{MHz}$ Project-X cavities for $\beta=0.6$ and $\beta=0.9$ are shown in Table 5 . The full cavity is made of five cells. Attached to the both sides of the cavity is the beam pipe of the radius $r$ from Table 5. Length of the beam pipe is a variable parameter in field calculations.

Results of spectrum calculation of $650 \mathrm{MHz}$ cavities using SuperLANS code and PEC boundary conditions at the beam pipe ends are shown in Figure 12 for non-propagating monopole modes and in Figure 13 for dipole HOMs. Figure 14 and Figure 15 show impedances of the same modes. ${ }^{6}$ One can observe, that all monopole HOMs in LE $650 \mathrm{MHz}$ section have impedance

${ }^{6}$ We use the following definition of the dipole mode impedance:

$$
\left(\frac{R}{Q}\right)^{(1)}=\frac{\left.\left|\int\left(\nabla_{\perp} E_{z}\right)\right|_{x=x_{0}} e^{i \omega z / v} d z\right|^{2}}{W \omega},
$$

where $\omega$ is the mode circular frequency, $W$ is stored energy, $v$ is beam velocity. The integral is taken along the line parallel to the cavity axis at the distance $x_{0}$. 


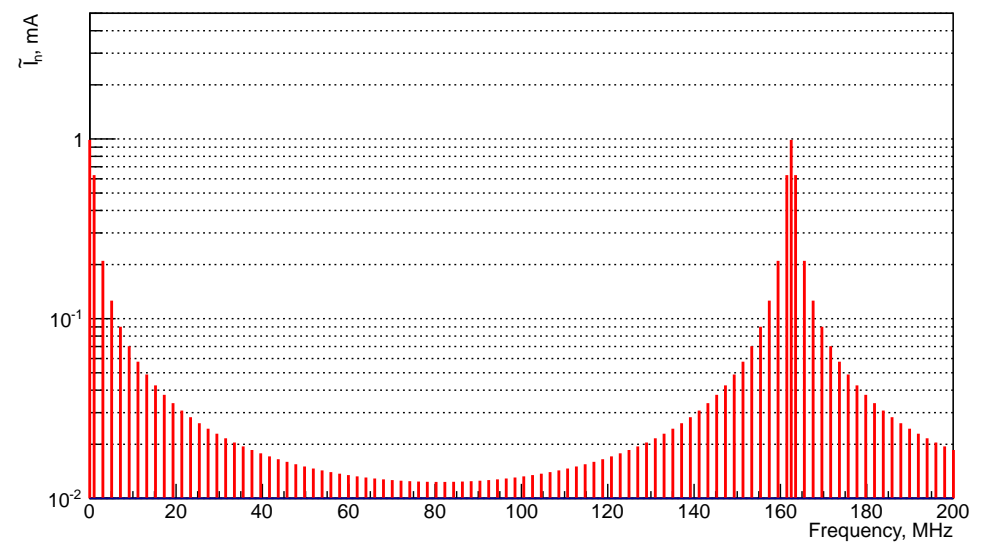

Figure 10: Beam spectrum of Muon Collider pulses.

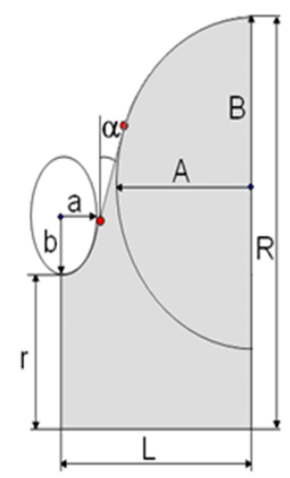

Figure 11: Parameterization of geometry of elliptical cavities. line 
Table 5: Geometrical parameters of $650 \mathrm{MHz}$ Project-X cavities.

\begin{tabular}{|c||c|c||c|c|}
\hline \multicolumn{1}{|c||}{} & \multicolumn{2}{c||}{$\beta_{G}=0.61$} & \multicolumn{2}{c|}{$\beta_{G}=0.9$} \\
\cline { 2 - 5 } Dimension & Regular Cell & End Cell & Regular Cell & End Cell \\
\hline$r, \mathrm{~mm}$ & 41.5 & 41.5 & 50 & 50 \\
$R, \mathrm{~mm}$ & 195 & 195 & 200.3 & 200.3 \\
$L, \mathrm{~mm}$ & 70.3 & 71.4 & 103.8 & 107.0 \\
$A, \mathrm{~mm}$ & 54 & 54 & 82.5 & 82.5 \\
$B, \mathrm{~mm}$ & 58 & 58 & 84 & 84.5 \\
$a, \mathrm{~mm}$ & 14 & 14 & 18 & 20 \\
$b, \mathrm{~mm}$ & 25 & 25 & 38 & 39.5 \\
$\alpha,{ }^{\circ}$ & 2 & 2.7 & 5.2 & 7 \\
\hline
\end{tabular}

946.6, 950.3, 1376 and $1383 \mathrm{MHz})$ with $(R / Q)^{(1)}>10^{4} \Omega / \mathrm{m}^{2}$.

Since for a non-relativistic beam HOM impedance depends on beam velocity, we also calculated $(R / Q)$ as a function of $\beta$. Figure 16 shows dependence of impedance on the beam velocity for the potentially dangerous monopole and dipole modes in $\mathrm{HE} 650 \mathrm{MHz}$ cavities.

We will perform measurements of HOM spectra and impedances of 650 $\mathrm{MHz}$ cavities and compare them to the calculated results when 5-cell prototype cavities will become available at Fermilab later this year.

\section{2. $H W R$ and $S S R$ cavities}

The spectra of HWR and SSR cavities are quite sparse. The HOM impedances fall quickly with frequency and are of the order of few $\mathrm{m} \Omega$ at frequencies above $2 \mathrm{GHz}$. We do not expect large HOM losses in these sections of the linac.

An extensive HOM analysis of SSR1 cavities including simulation of spectra and impedances of monopole, dipole, and quadruple modes as well as measurements of spectra and $R / Q$ values of monopole HOMs up to $2 \mathrm{GHz}$ of six fabricated SSR1 resonators has been performed and presented in [13]. The calculated and measured values are found to be in a good agreement. The measured HOM frequency spread is within $7 \mathrm{MHz}$.

Simulation studies of SSR2 and HWR cavities, similar to these of SSR1 described above, have been started. We will report results soon. 


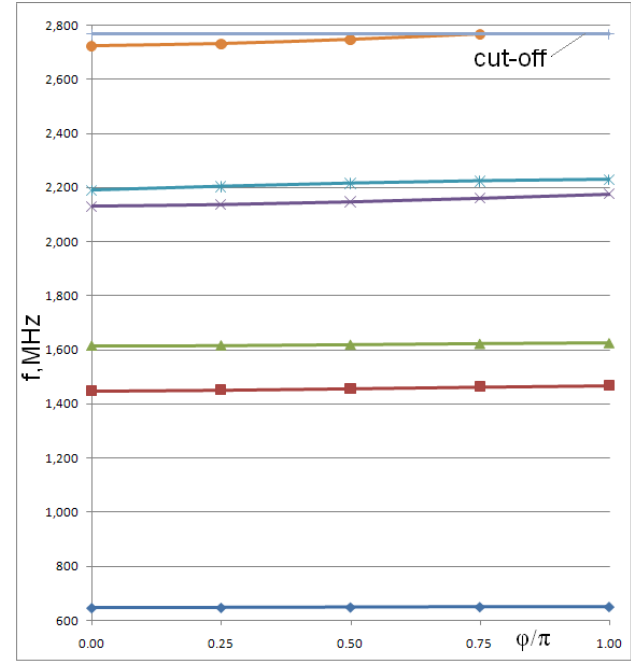

a)

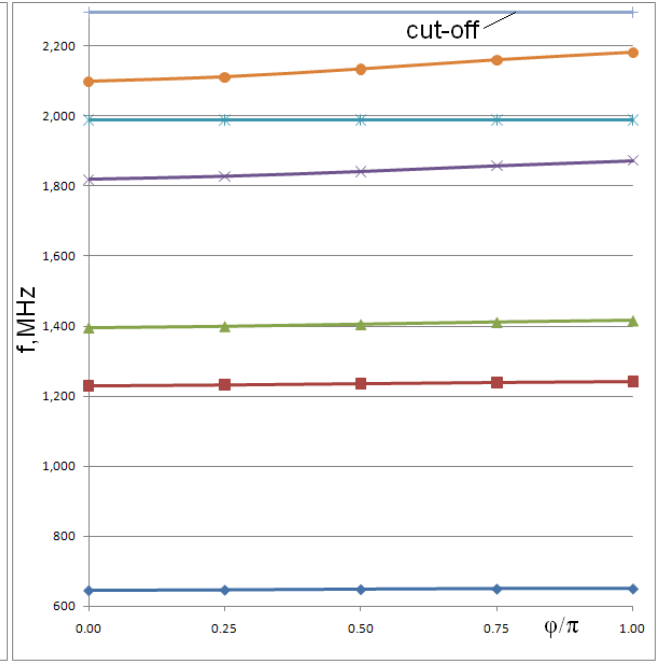

b)

Figure 12: Spectrum of monopole HOMs in LE 650 (a) and HE 650 (b) sections of Project$\mathrm{X}$ linac.

\section{Resonance HOM excitation}

A bunched continuous beam passing through a superconducting cavity may coherently excite cavity HOMs with high $Q$-factor, which are close to one of the beam spectrum lines. Here we present an estimations for the resonance excitation of the monopole and dipole high order modes in the HE part of the Project-X linac.

We require, that the monopole modes should not increase the longitudinal emittance of the beam, $\varepsilon_{z}$ :

$$
\sigma_{U_{H O M}} \sigma_{z} / c \ll \varepsilon_{z},
$$

where $\sigma_{U_{H O M}}=\frac{1}{\sqrt{2}} \hat{U}_{H O M}$ is the r.m.s. of energy gain caused by HOM with the amplitude $\hat{U}_{H O M}, \sigma_{z}$ is a bunch length, and $c$ is speed of light. For high- $Q$ resonances one can get: 8

$$
\hat{U}_{H O M} \approx \frac{\tilde{I}(R / Q)}{4 \delta f / f},
$$

\footnotetext{
${ }^{8}$ We use the following exact expression for the amplitude of HOM with frequency $f$ and impedance $(R / Q)$ excited by beam harmonic with amplitude $\tilde{I}$ and frequency $f+\delta f$
} 


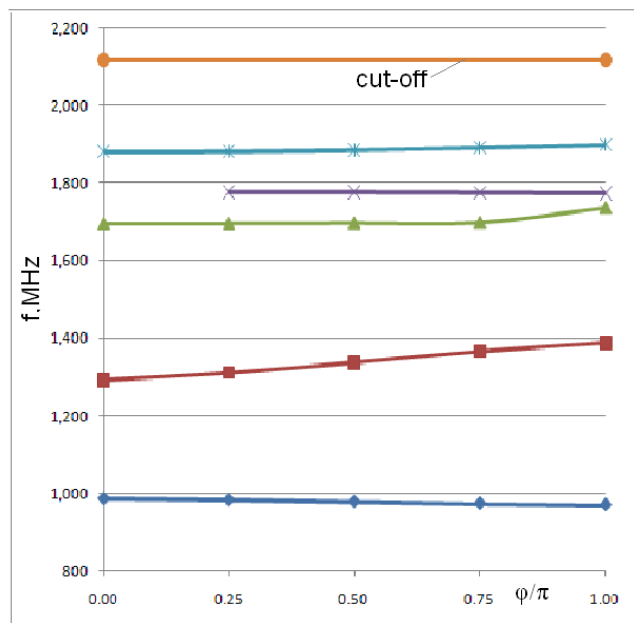

a)

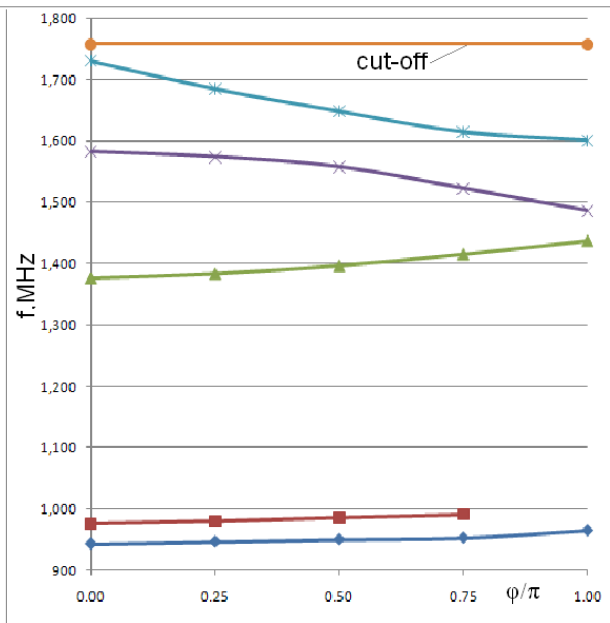

b)

Figure 13: Spectrum of dipole HOMs in LE 650 (a) and HE 650 (b) sections of Project-X linac.

where $\tilde{I}$ is the amplitude of a beam spectrum line, $\delta f$ is the difference between the HOM frequency $f$ and the beam spectrum line frequency. Here we assume that $\delta f / f \gg 1 / Q$.

Finally, we obtain an estimation for the frequency detuning, $\delta f$, that does not lead to a longitudinal emittance growth:

$$
\delta f \gg f \frac{\tilde{I}(R / Q) \sigma_{z}}{4 \sqrt{2} \varepsilon_{z} c} .
$$

We have the worst case at the start of the $\mathrm{HE} 650 \mathrm{MHz}$ section, where the bunch length is at the maximum $\left(\sigma_{z} / c=7.7 \times 10^{-3} \mathrm{~ns}\right)$, the second passband monopole HOM $(1241 \mathrm{MHz}$ and highest $R / Q=130 \Omega$ ), the nearest large amplitude beam spectrum line $(\tilde{I}=1 \mathrm{~mA})$, and the results from Eq. 3: $\delta f \gg 140 \mathrm{~Hz}$, obtained with the value of the emittance $\varepsilon_{z}=1.5 \mathrm{keV} \cdot \mathrm{ns}$ [15]. Thus, if the distance between the beam spectrum lines is $1 \mathrm{MHz}$ and the

(see, for example, [14]):

$$
\hat{U}_{H O M}=-i \frac{f(f+\delta f)}{(f+\delta f)^{2}-f^{2}-i \frac{f(f+\delta f)}{Q}} \frac{\tilde{I}}{2}\left(\frac{R}{Q}\right) .
$$




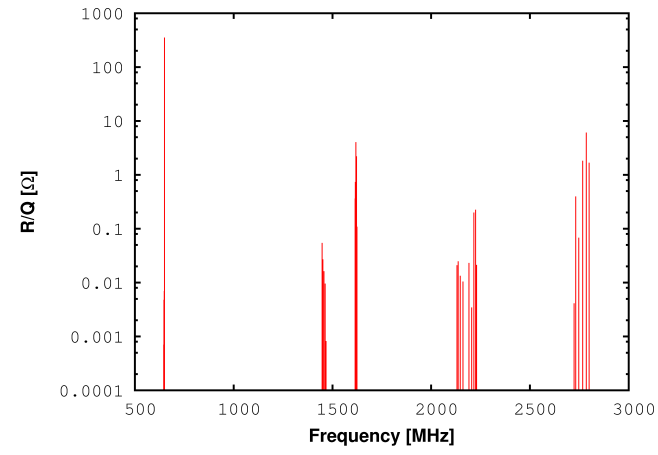

a)

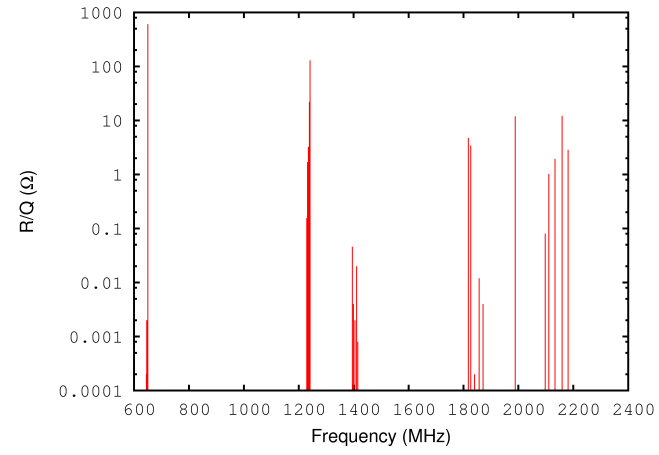

b)

Figure 14: Impedance of monopole HOMs in LE 650 (a) and HE 650 (b) sections of Project-X linac.

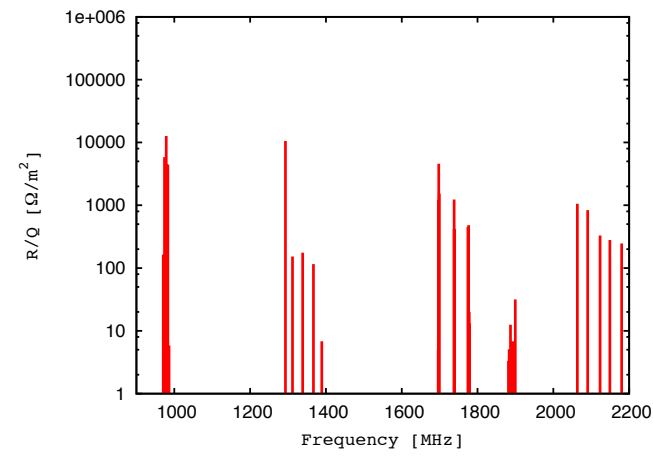

a)

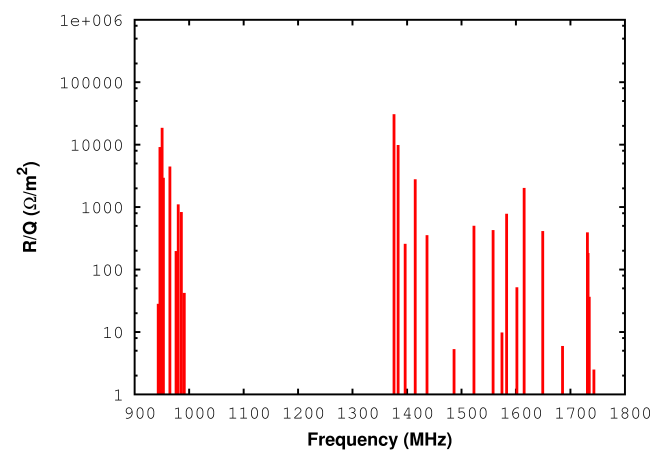

b)

Figure 15: Impedance of dipole HOMs in LE 650 (a) and HE 650 (b) sections of Project-X linac.

monopole $\mathrm{HOM}$ frequency spread is about $1-2 \mathrm{MHz}$, the probability that the cavity has the resonant frequency close enough to the beam spectrum line is less than $10^{-4}$ for the baseline of the ProjectX CW linac.

The cryogenic losses are proportional to the square of the HOM field amplitude:

$$
P_{\text {loss }} \approx \frac{\hat{U}_{H O M}^{2}}{(R / Q) Q_{0}},
$$

where $Q_{0}$ is an intrinsic quality factor. Therefore, it requires much close proximity of the beam spectrum line and HOM frequency in order to get significant cryogenic losses. If the HOM mode is exactly at the resonance, $U_{H O M}=\frac{1}{2} \tilde{I}(R / Q) Q_{L}$, where $Q_{L}$ is the loaded quality factor of the mode. 


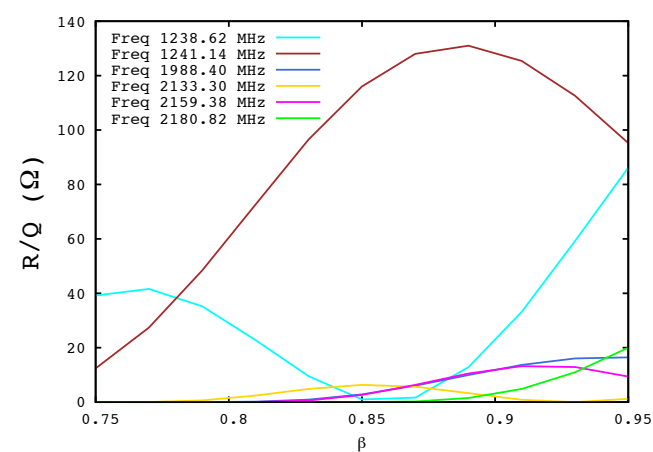

a)

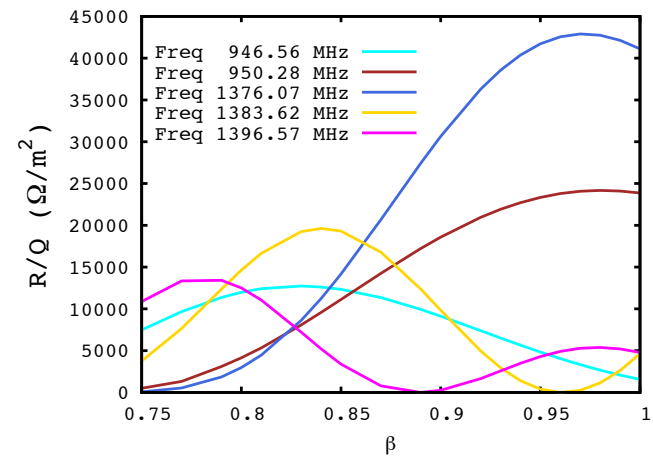

b)

Figure 16: Impedance of the most dangerous monopole (a) and dipole (b) HOMs as a function of beam velocity $\beta$ in $\mathrm{HE} 650 \mathrm{MHz}$ cavities.

Requiring that $P_{\text {loss }}$ is much smaller than the sum of the static heat load and the cryogenic losses due to accelerating mode $(\sim 20 \mathrm{~W})$, and assuming that the intrinsic quality factor is $Q_{0}=5 \times 10^{9}$, we estimate that the maximum allowable value of the monopole HOM loaded quality factor is $Q_{L} \ll 6 \times 10^{7}$.

A similar approach can be employed for the analysis of dipole modes resonance excitation. The transverse kick caused by an HOM is:

$$
U_{k i c k} \approx \frac{f}{4 \delta f}\left(\frac{x_{0}}{k}\right) \tilde{I}(R / Q)^{(1)},
$$

where $x_{0}$ is the transverse offset of the beam trajectory, $k=2 \pi / \lambda$ is the wave number, and $(R / Q)^{(1)}$ is the transverse impedance (see definition in Section 4.1). Transverse emittance increase $\delta \varepsilon_{t}$ may be estimated in the following way:

$$
\delta \varepsilon_{t} \approx \Delta x^{\prime} \sigma_{x}=\frac{U_{k i c k}}{\sqrt{2} p_{\| c}} \sqrt{\varepsilon_{t} \beta_{f}},
$$

where $\varepsilon_{t}=2.5 \times 10^{-7} / \beta \gamma \mathrm{m}$ is the beam transverse emittance [15], and $\beta_{f}$ is a beta-function near the cavity. Finally, we can rewrite:

$$
\delta f \gg \frac{c x_{0} \tilde{I}(R / Q)^{(1)}}{8 \sqrt{2} \pi \beta \gamma U_{0} \sqrt{\varepsilon_{t} / \beta_{f}}},
$$

where $U_{0}$ is the proton mass in $\mathrm{eV}$. The worst case corresponds to the beginning of $\mathrm{HE} 650 \mathrm{MHz}$ section of Project-X linac, where the dipole mode has highest transverse shunt impedance $\left(f=1376 \mathrm{MHz},(R / Q)^{(1)}=60 \mathrm{k} \Omega / \mathrm{m}^{2}\right)$, 
proton energy is $500 \mathrm{MeV}\left(\beta_{f}=15 \mathrm{~m}\right)$, the beam offset is $1 \mathrm{~mm}$, the nearest beam spectrum line amplitude is $0.3 \mathrm{~mA}$, and the resulting frequency detuning is $\delta f \gg 1 \mathrm{~Hz}$. When a dipole HOM is in exact resonance,

$$
U_{k i c k}=\frac{x_{0}}{k} \frac{\tilde{I}}{2}(R / Q)^{(1)} Q_{L}
$$

Using this and Eq. 6 we estimate the maximum allowable value of the dipole HOM loaded quality factor to be $Q_{L} \ll 6 \times 10^{8}$. Typical value of $Q_{L}$ in superconducting RF cavities is less (much less for the most HOMs) than $10^{8}$. Also, condition on $\delta f$ can be satisfied by cavity spectrum manipulation (see Section 6). Therefore, the growth of the beam transverse emittance does not look to be a problem for the Project-X linac.
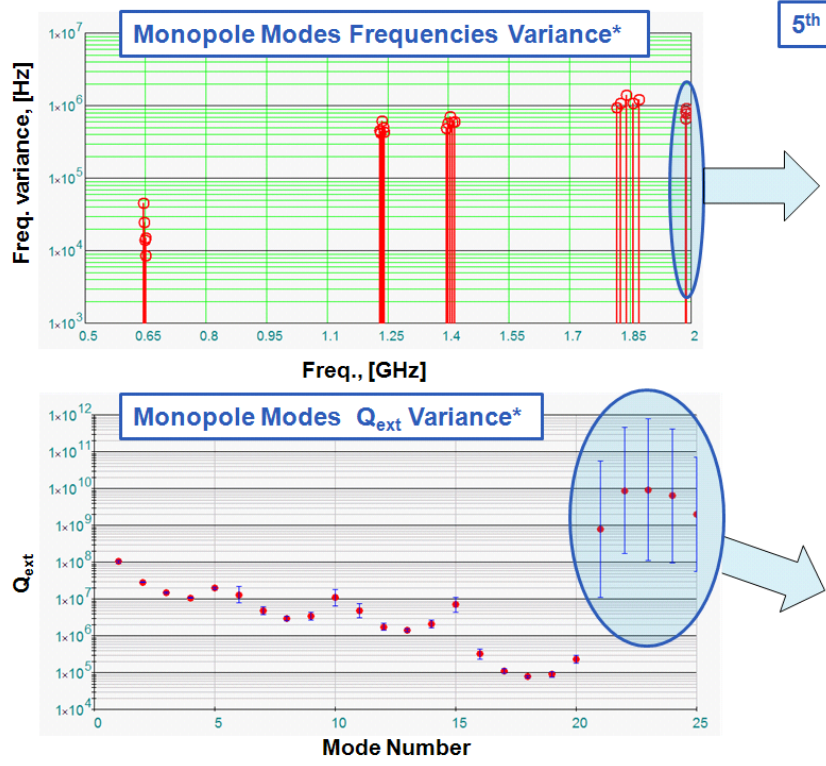

* $650 \mathrm{MHz}$, beta $=0.90$ Project $X$ structure with $\pm 0.2 \mathrm{~mm}$ tolerance applied

$5^{\text {th }}$ Monopole Passband Histograms*
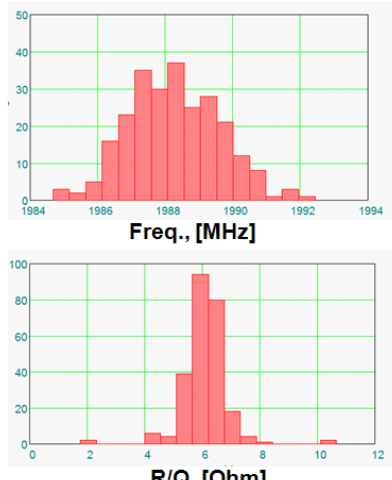

$\mathrm{R} / \mathrm{Q},[\mathrm{Ohm}]$

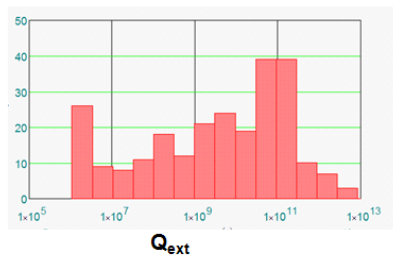

Figure 17: Variations of monopole HOMs parameters for the HE $650 \mathrm{MHz}$ Project-X structure.

In order to accurately estimate the probability of resonance HOM excitation in the Project-X linac by one of the beam component, one has to do statistical analysis, which requires spread of the data for HOM parameters (frequency, impedance and quality factor). For the ILC cavity the r.m.s. spread $\sigma_{f}$ of the resonance frequency is about $6-9 \mathrm{MHz}$ depending on the 


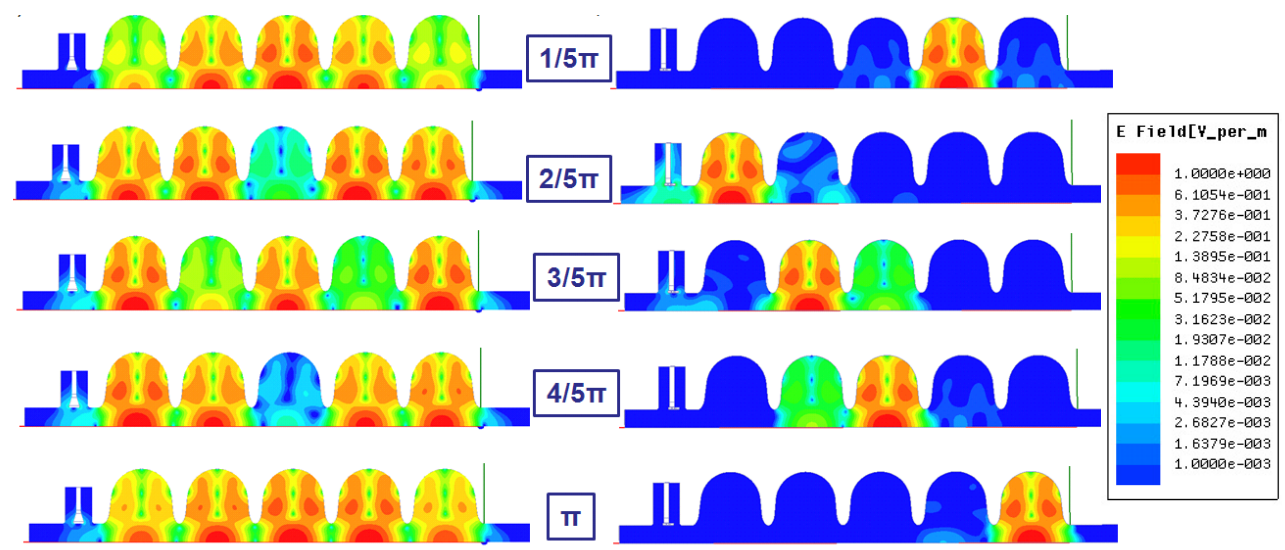

a)

b)

Figure 18: Electric fields distortion of the $5^{\text {th }}$ monopole band for the $\mathrm{HE} 650 \mathrm{MHz}$ Project$\mathrm{X}$ structure; a) an ideal structure, b) a structure in presence of misalignments. In both cases the operating mode is tuned to correct frequency and field flatness.

pass-band, according to the DESY measurement statistics [16]. We do not have the experimental data available for Project-X linac yet. One way to obtain this information is to perform cavity HOM simulations taking into account manufacturing mechanical tolerances. Measurements of the profile of $1.3 \mathrm{GHz}$ ILC cavity made in Cornell show that there are deviations from the ideal cavity of the order of $\pm 0.2 \mathrm{~mm}$ [17]. We take this value as a base for our statistical simulation of the Project-X HE $650 \mathrm{MHz}$ structure. Geometrical parameters of the cavity individual half-cells (as shown in Table 5) allowed to randomly vary withint $\pm 0.2 \mathrm{~mm}$ tolerances around standard values. While running the simulation with an imperfect cavity geometry, we tune the individual cell frequency by changing its length and preserving the field flatness of the accelerating mode along the cavity at the same time. Finally, we calculate HOM spectrum of the derived 5-cell structure, using HFSS code [18] with PEC boundary conditions at the beam pipe ends. Data are accumulated for further statistical analysis by repeating the simulation for multiple random structures. A typical result for the variations of monopole HOMs parameters is illustrated in Figure 17.

Besides the natural spread of the HOM parameters from cavity to cavity, random deviations of geometry from the designed shape due manufacturing tolerances in real cavity, may significantly change EM field distribution of 

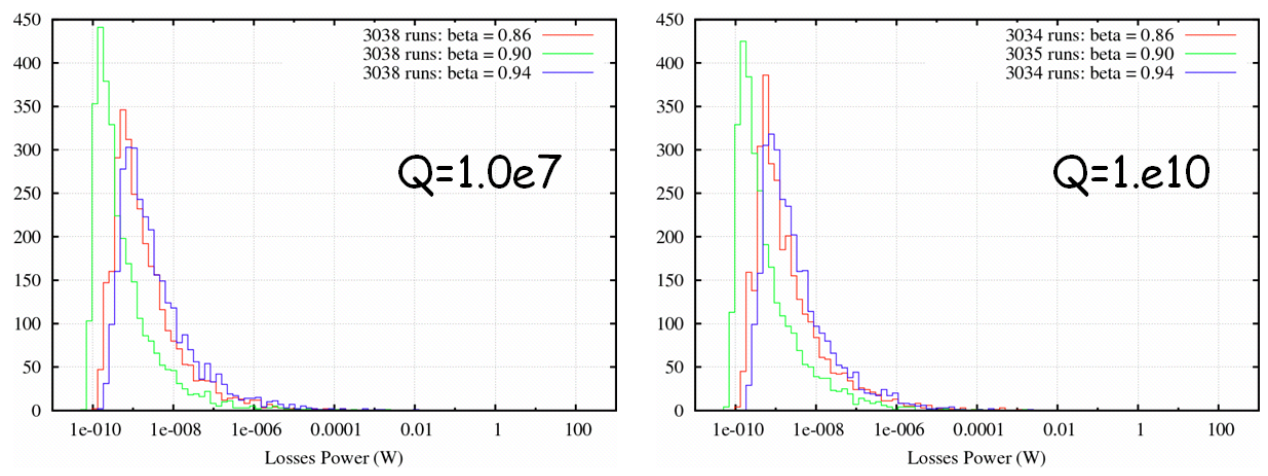

Figure 19: Calculations of the power losses in the HE $650 \mathrm{MHz}$ cavities.

HOMs from a very narrow pass-band, when cell-to-cell coupling is very weak. ${ }^{9}$ This effect is illustrated in Figure 18 for the $5^{\text {th }}$ monopole pass-band of the HE $650 \mathrm{MHz}$ Project-X cavity. Some of the modes, such as $\pi / 5,3 \pi / 5$, and $4 \pi / 5$ in Figure $18 \mathrm{~b}$, become trapped in the internal cells of a cavity. Such a distortion causes $(R / Q)$ and $Q_{L}$ of an actual cavity to differ significantly from theoretical values.

By using the predicted deviations of monopole HOMs frequency, $Q_{L}$, and $(R / Q)$ we generated $10^{5}$ random linacs in order to estimate probability of the RF losses per cryomodule. The results of this computation for the HE $650 \mathrm{MHz}$ cavities are presented in Figure 19. The tail of these distributions in the region of a high power losses has only a weak dependence on the beam energy. Thus, we can neglect the effect of RF losses variation along the linac. The cumulative probability of losses per cryomodule is shown in Figure 20 for three cases, when maximum, theoretical and actual $(R / Q)$ values are taken into account. We can see from this plot, that the probability to have losses of $1 \mathrm{~W}$ is in the range $3 \times 10^{-5}-10^{-3}$; or, alternatively, the HOM power loss is in the range $0.01-1 \mathrm{~W}$ per cryomodule with a probability $10^{-3}$. Our simulation also shows that main contribution in the region of high losses is due to trapped modes of the $5^{t h}$ pass-band.

\footnotetext{
${ }^{9}$ In this case, when geometry of individual cells vary across the cavity, coupling between cells is weak and also varies from cell to cell, the usual pass-band structure of $N$ modes of $m \pi / N$-kind, where $N$ is number of cells and $m$ runs from 1 to $N$ ([2], Chapter 7 ), may change. The field of a mode may, for example, be concentrated in a single cell, or two adjacent cells.
} 


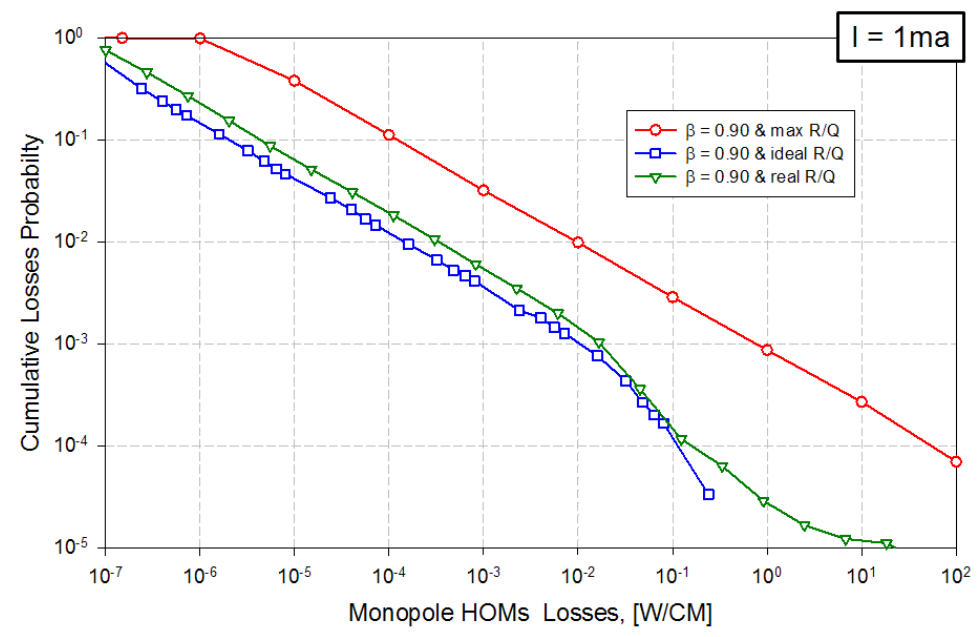

Figure 20: Probability of monopole HOMs power loss per cryomodule for the HE 650 $\mathrm{MHz}$ cavities of the Project-X linac; red is maximum $(R / Q)$ w.r.t. the beam velocity for all HOMs; blue is theoretical $(R / Q)$ for ideal cavity, and green is "realistic" $(R / Q)$ for a cavity with mechanical errors.

We may conclude that cryogenic losses due to resonant excitation of monopole $\mathrm{HOM}$ in $\mathrm{HE} 650 \mathrm{MHz}$ structures is not a problem for the considered Project-X parameters, CW regime and $1 \mathrm{~mA}$ average beam current.

Operation of Project-X linac in a high beam current regime. Possible future upgrades of Project-X may require to operate linac in a regime of a high beam current, when an average beam current is $5 \mathrm{~mA}$ or even $10 \mathrm{~mA}$. As it has been discussed earlier in this section, monopole high order modes of the $5^{\text {th }}$ pass-band of $\mathrm{HE} 650 \mathrm{MHz}$ structure may become trapped in the internal cells of the cavity, leading to a high value of $Q_{L}$. This and the fact that cryogenic losses are proportional to square of the average beam current, $P_{\text {loss }} \sim I_{\text {beam }}^{2}$ may potentially increase $P_{\text {cryo }}$ up to hundred Watt for the $10 \mathrm{~mA}$ average beam current.

Figure 21 shows dependence of bandwidth of the $5^{\text {th }}$ passband on geometrical beta (a) and aperture (b). An alternative design of elliptical cavity with a larger aperture $\left(59 \mathrm{~mm}\right.$ ) and $\beta_{G}=0.92$ is suggested for $\mathrm{HE} 650 \mathrm{MHz}$ section in order to comply with various upgrade scenarios of the Project $\mathrm{X}$ linac [19]. Geometrical parameters of the cavity are shown in Table 6. We perform a similar statistical analysis of the HOM spectrum for this cavity. Figure 22 shows monopole HOM RF losses for the beam current of $1 \mathrm{~mA}$ and 
Table 6: Geometrical parameters of alternative cavity design for $\mathrm{HE} 650 \mathrm{MHz}$ section of Project X linac.

\begin{tabular}{|c||c|c|}
\hline \multirow{2}{*}{\multicolumn{1}{|c||}{ Dimension }} & \multicolumn{2}{c|}{$\beta_{G}=0.92$} \\
\cline { 2 - 3 } & Regular Cell & End Cell \\
\hline$r, \mathrm{~mm}$ & 59 & 59 \\
$R, \mathrm{~mm}$ & 200.05 & 200.05 \\
$L, \mathrm{~mm}$ & 97.56 & 106.08 \\
$A, \mathrm{~mm}$ & 84 & 85 \\
$B, \mathrm{~mm}$ & 90 & 78 \\
$a, \mathrm{~mm}$ & 13 & 20 \\
$b, \mathrm{~mm}$ & 28 & 33 \\
$\alpha{ }^{\circ}$ & 1.3 & 1.9 \\
\hline
\end{tabular}

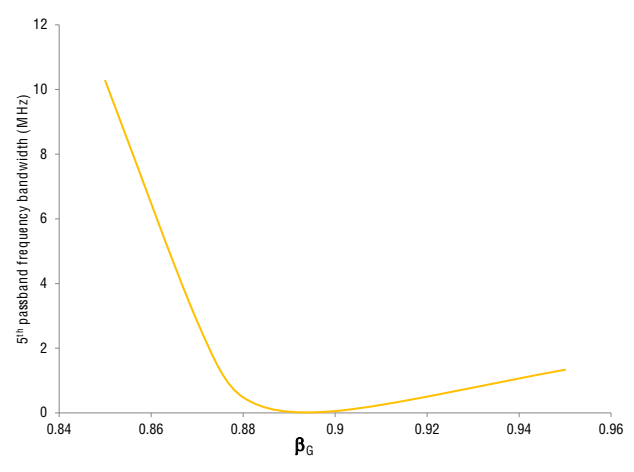

a)

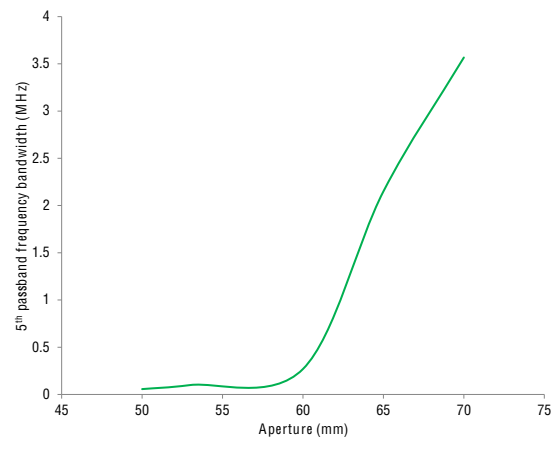

b)

Figure 21: Dependence of the $5^{t h}$ pass-band bandwidth on geometrical beta, $\beta_{G}$ (a) and aperture (b). 

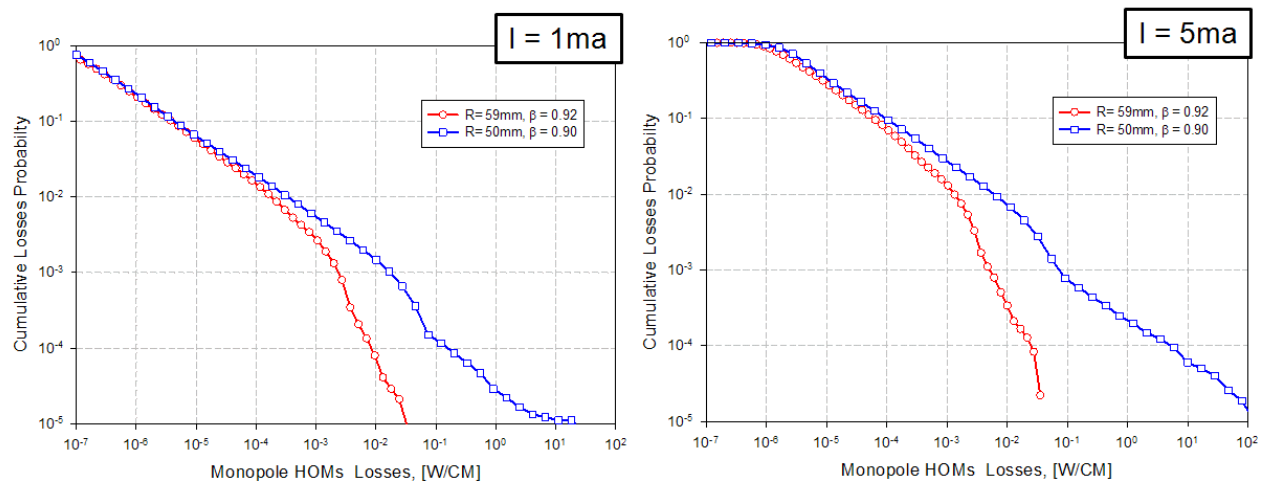

Figure 22: Probability of monopole HOMs RF losses per cryomodule for the present (blue) and alternative (red) version of $\mathrm{HE} 650 \mathrm{MHz}$ cavities for Project-X.

$5 \mathrm{~mA}$ for both present and alternative cavities. The new cavity shape suppresses high $Q_{L}$ of the monopole HOM completely and mitigates the problem of large cryogenic losses.

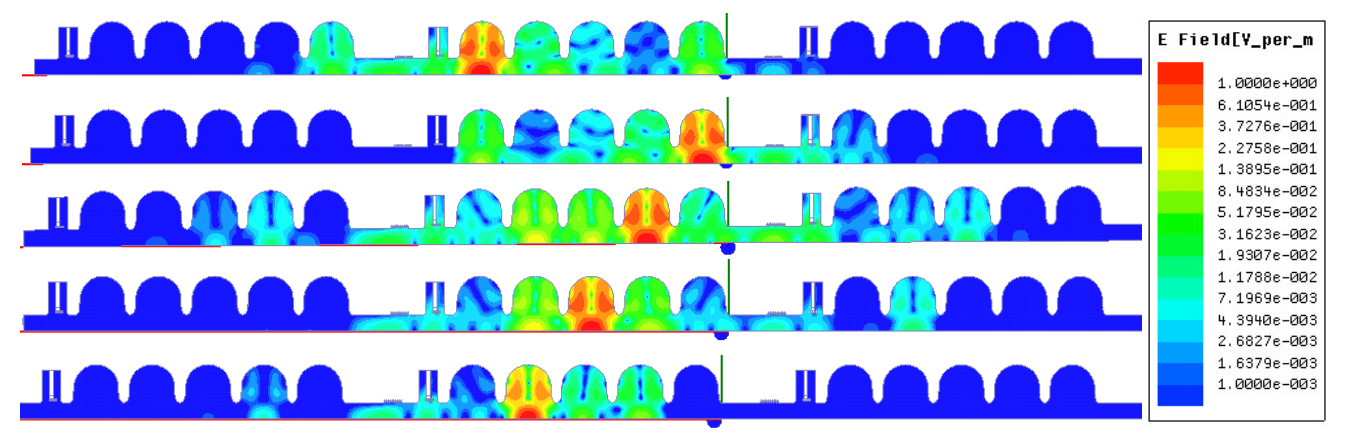

Figure 23: Field distribution for propagating monopole HOMs that are effectively trapped inside the cavity chain.

It is interesting to note, that in the alternative $\mathrm{HE} 650 \mathrm{MHz}$ cavity with a larger aperture, monopole modes of the $5^{\text {th }}$ pass-band have frequencies above the beam pipe cut-off frequency and become propagating modes. We perform simulation of RF field in a chain of three cavities interconnected with bellows, randomly varying geometry of cavities, as described above. We find solutions of our simulation where the field of the propagating HOMs is concentrated in the central cavity and the modes are effectively trapped inside the cavity chain. Figure 23 shows distribution of RF fields of trapped 


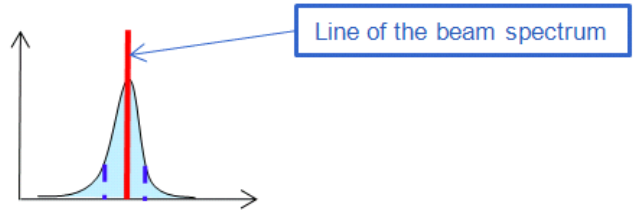

a)

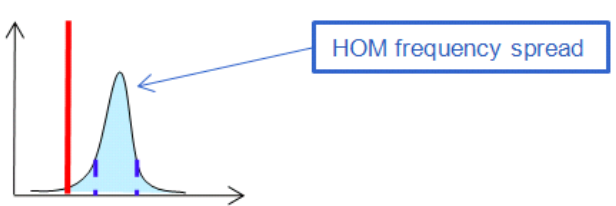

b)

Figure 25: HOM excitation by beam sub-harmonic; a) - symmetrical and b) non - symmetrical options. 


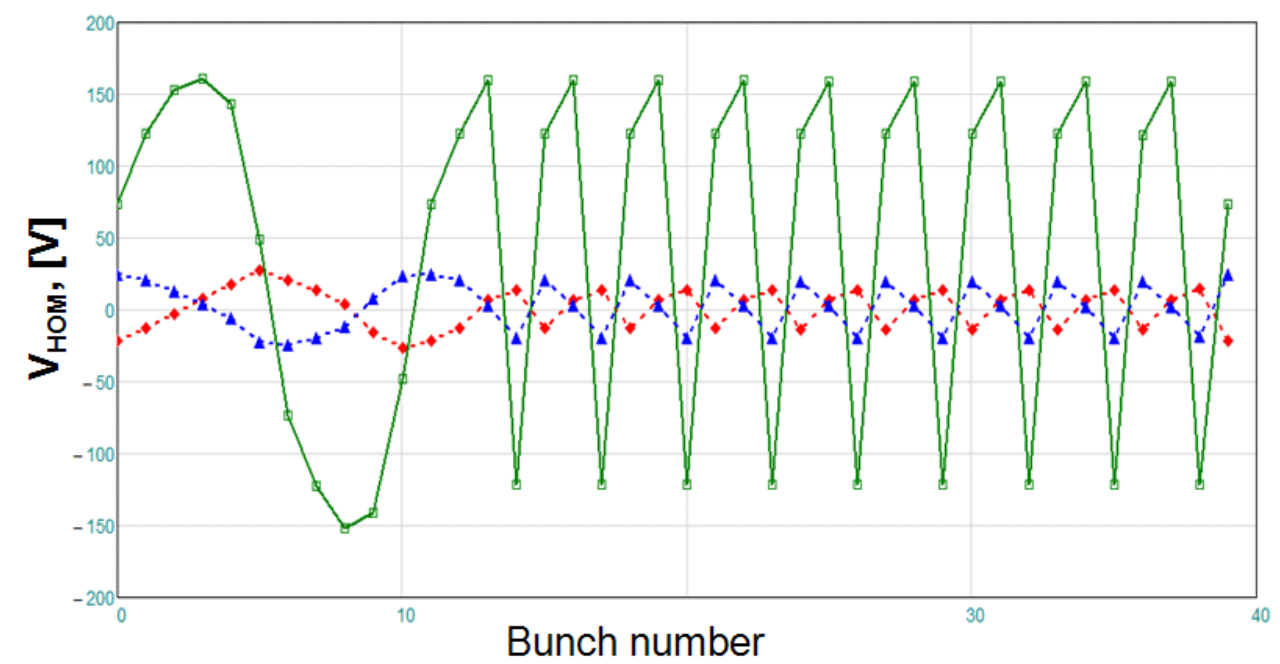

Figure 26: Monopole HOM $\left(I_{\text {beam }}=1 \mathrm{~mA}, Q_{L}=10^{5}\right)$ excitation by the $10 \mathrm{MHz}$ beam component, green curve - on resonance, read and blue - of resonance $( \pm 3 \sigma)$ excitations.

The width of a beam spectrum line is much more narrow compared to the spread of HOM frequencies. We consider two possible situations, when a beam line frequency is close to an HOM frequency:

1. symmetric, shown in Figure 25a, when a line in the beam spectrum coincides with the mean value of the HOM frequency distribution. By construction, this is unlikely situation. Energy spread along the bunch train does not increase as beam travels along linac.

2. non-symmetric, when the beam line frequency is shifted with respect to the HOM frequency (see Figure 25b). This is very probable situation. The energy spread caused by the HOMs excitation accumulates along the linac.

Figure 26 illustrates the principle of compensation (or accumulation) of the beam energy spread. The typical process of the longitudinal emittance growth is shown on Figure 27.

We perform a statistical analysis in order to calculate the probability of the beam longitudinal emittance growth as the following. We simulate $10^{5}$ Project X linacs (HE $650 \mathrm{MHz}$ section only) with random variation of $\mathrm{HOM}$ parameters (frequency, impedance, loaded quality factor ) in cavities according to the model described earlier in this Section (see Figure 17). Energy 


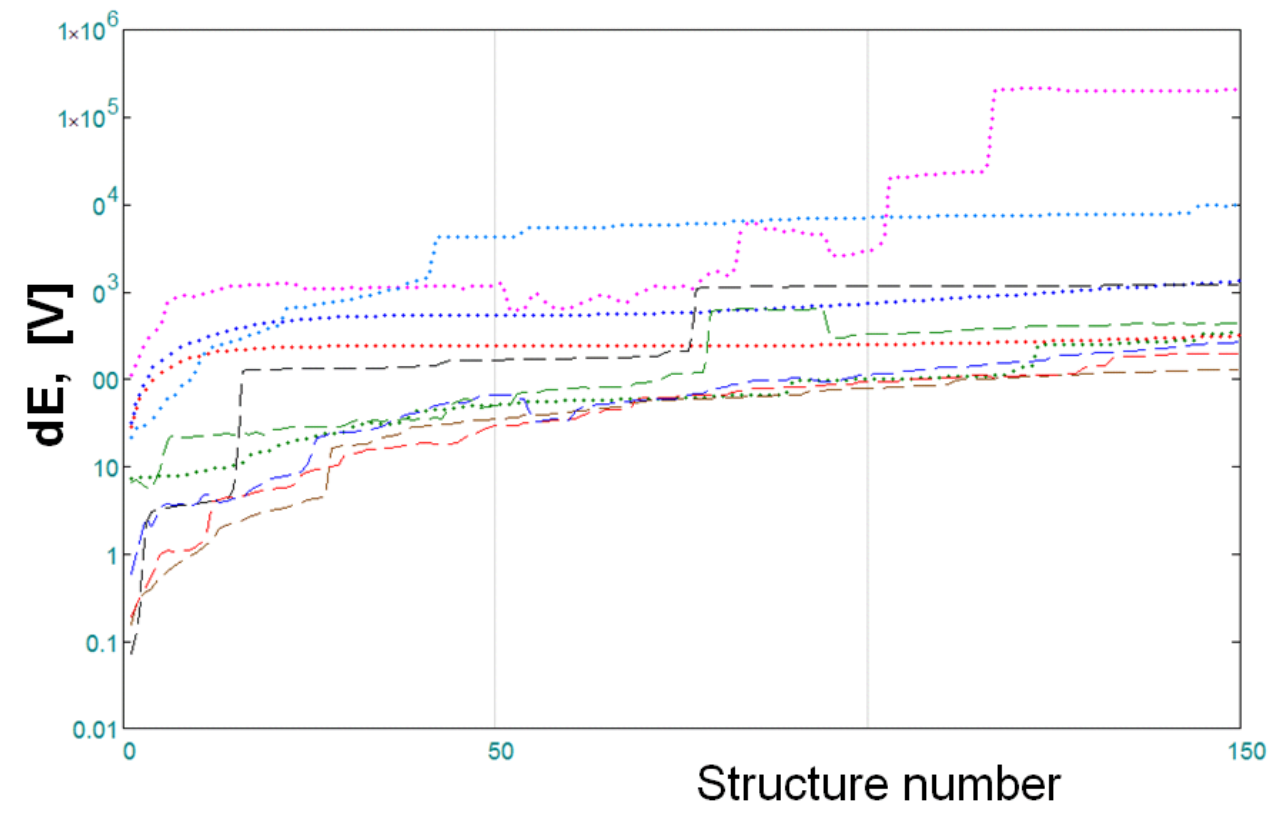

Figure 27: Growth of the energy spread in the beam train along the $\mathrm{HE} 650 \mathrm{MHz}$ part of Project-X linac due to monopole HOMs excitations; dotted curves $-1^{\text {st }}$ and $2^{\text {nd }}$ passbands, dashed $-5^{\text {th }}$ passband.

spread from bunch to bunch and longitudinal emittance growth are determined for every simulated linac. The results are summarized in Figure 28 and Figure 29 for $1 \mathrm{~mA}, 5 \mathrm{~mA}$ and $10 \mathrm{~mA}$ average beam currents. The simulation shows that the $2^{\text {nd }}$ and $5^{\text {th }}$ monopole passbands are the most dangerous and, thus, require a special care in order to avoid high- $Q$ resonances.

\section{Manipulation of HOM spectra}

It is shown in the previous section, that the probability of one of the $\mathrm{HOM}$ of $650 \mathrm{MHz}$ cavities to be in exact resonance with a line of beam current spectrum is small. In a rare case when it is happened, the following approach has been demonstrated to work [20]. HOM frequency can be moved by detuning cavity's accelerating mode by few tens of $\mathrm{kHz}$ and then tuning operating mode back to resonance.

A test, described in [20], was performed with $1.3 \mathrm{GHz}$ 9-cell ILC cavity cooled to $2 \mathrm{~K}$. The operating mode was detuned by $90 \mathrm{kHz}$ and then tuned back. Because of small residual deformation of the cavity, HOM frequencies 

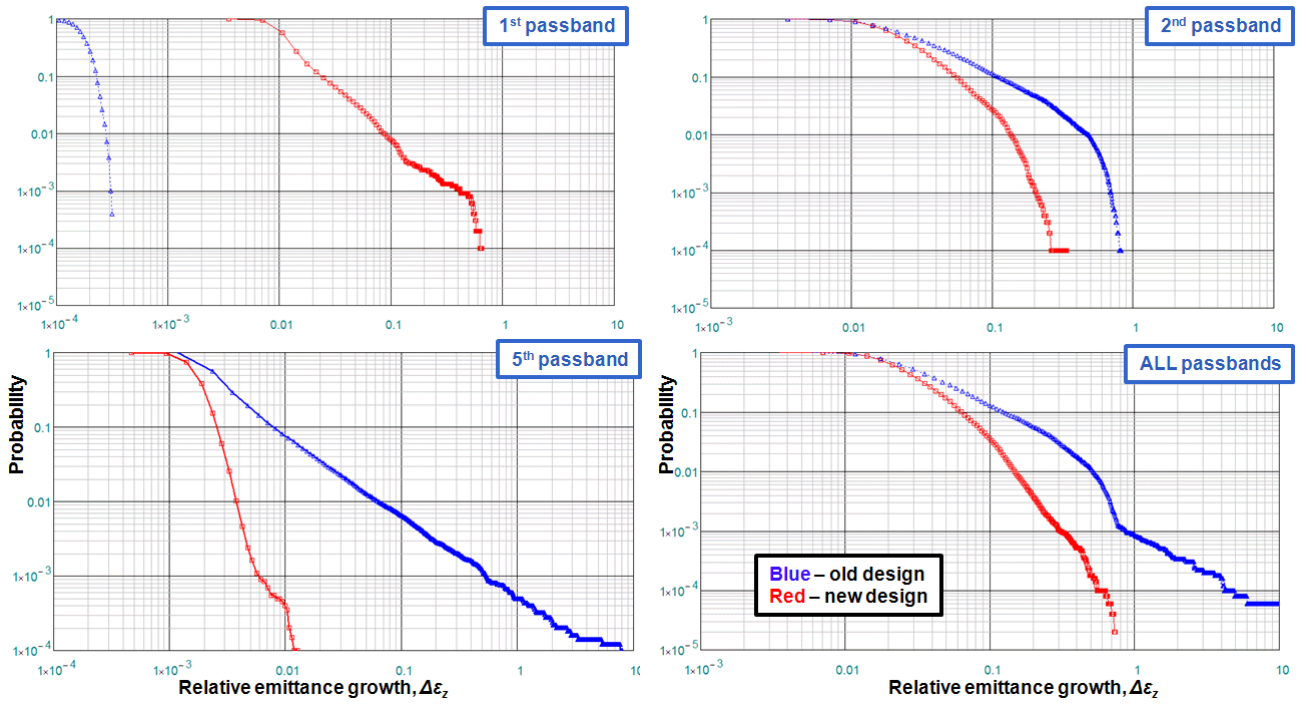

Figure 28: Probability of longitudinal emittance growth in the HE $650 \mathrm{MHz}$ part of the Project-X linac for $1 \mathrm{~mA}$ average beam current. Data for the present design of the cavity are shown in blue, data for the alternative design are in red.

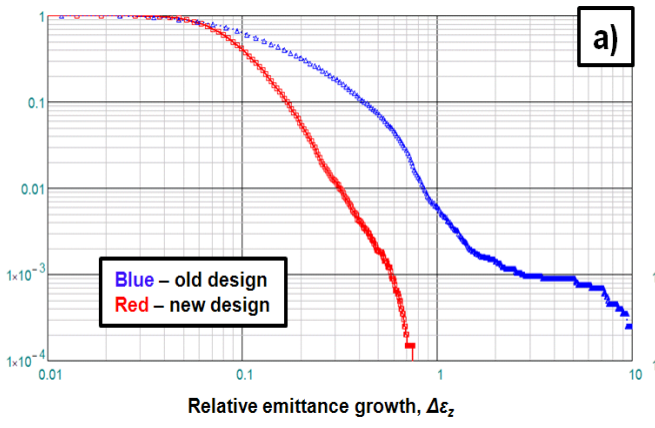

a)

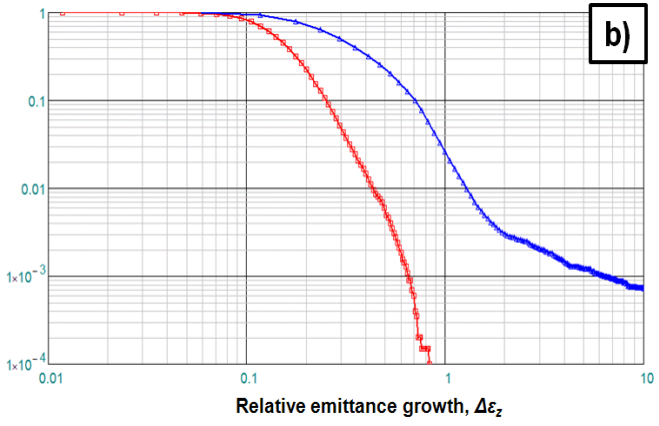

b)

Figure 29: Probability of longitudinal emittance growth in the $\mathrm{HE} 650 \mathrm{MHz}$ part of the Project-X linac for $5 \mathrm{~mA}$ (a) and $10 \mathrm{~mA}$ (b) average beam current. Data for the present design of the cavity are shown in blue, data for the alternative design are in red. 
moved by $100-500 \mathrm{~Hz}$ after this procedure.

\section{Collective beam effects}

We study two types of collective beam effects caused by HOMs: beam breakup, affecting transverse beam dynamics; and longitudinal, klystron-type instabilities (see, for example, [21, 22]). In general, we do not expect the collective effects to be an issue, due to the following: 1) there is no feedback in linac as it is in circular accelerators, each bunch interacts with every cavity only once, resonance and amplification conditions can not be realized as in circular accelerator when bunches pass that same cavities multiple times; 2) we have few different types of cavities with different spectra, HOMs excited in one type of cavities do not affect excitation of HOMs in other types of cavities, since frequencies of these modes are uncorrelated; 3) there is spread of HOM frequencies in each cavity type, caused by manufacturing errors, thus, even if a certain $\mathrm{HOM}$ is in resonance condition in one cavity, it may be off resonance in other cavities; 4) HOM impedances depend on beam velocity, amplitudes of excited HOMs vary from cavity to cavity and can only reach the maximum possible value in few cavities, where the beam velocity is optimal for HOM excitation; 5) beam current is small, power loss to HOMs scales as square of beam current (Equation (4)), effects of HOMs on longitudinal and transverse beam dynamic are proportional to the beam current (Equations (2) and (5)). We focus our study of beam instabilities on $650 \mathrm{MHz}$ section of Project X linac, the longest one with the highest beam velocity.

\subsection{Transverse dynamics}

We use a simplified model to investigate transverse beam dynamics in the field of dipole HOMs. We assume short bunches. We use lattice design as outlined in [23]. Five dipole passbands in LE and $\mathrm{HE} 650 \mathrm{MHz}$ section are taken into account. Misalignment of cavities is random. Beam timing structure corresponds to $3 \mathrm{GeV}$ program (Sec. 3, Figure 8). The deflecting gradient $U_{n+1}^{\prime}$ at the passage of bunch $n+1$ through a cavity can be written as the following:

$$
U_{n+1}^{\prime}=U_{n}^{\prime} e^{j \omega_{H O M} T-T / \tau}-\frac{1}{2} q_{b} R^{(1)} \omega_{H O M}\left(x-x_{c a v}\right),
$$

where $q_{b}$ is the bunch charge, $R^{(1)}=Q_{e x t}(R / Q)^{(1)}$ is the dipole mode impedance, $x$ and $x_{c a v}$ are the beam and cavity offsets. Then, change of the bunch 


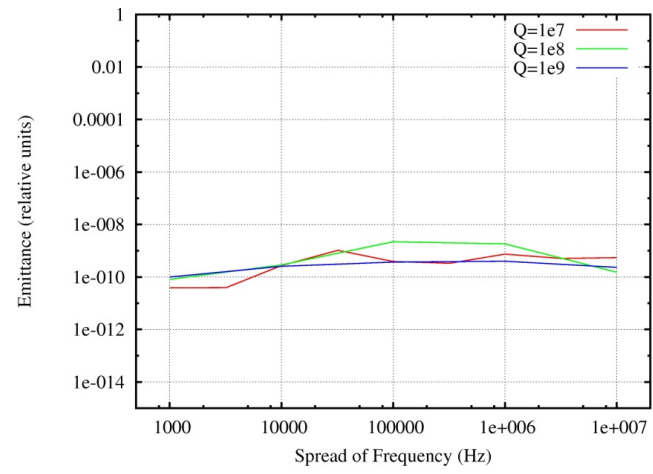

a)

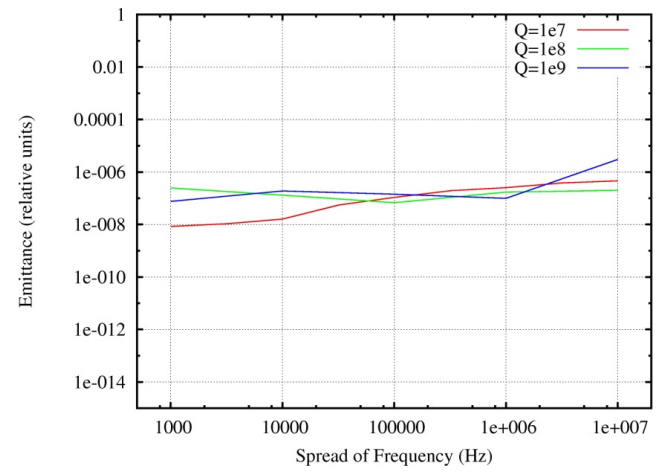

b)

Figure 30: Transverse (a) and longitudinal (b) emittance dilution as a function of HOM frequency spread.

transverse momentum is

$$
\Delta p_{\perp}=\Re\left(j \frac{\beta}{\omega} U^{\prime}\right)
$$

We run simulation for $1 \mathrm{~mA}$ average beam current and $0.5 \mathrm{~mm}$ R.M.S. of random transverse offsets of cavities. Results of simulation are shown in Figure 30 (left). One can see, that the variations of the transverse emittance are negligible in the the wide range of HOM frequency spread and $Q_{\text {ext }}$.

\subsection{Longitudinal instability}

Similarly, we estimate effect of longitudinal HOMs on the longitudinal beam dynamics (the so-called "klystron-type" instability). Figure 30 (right) shows results of this simulation. Again, no noticeable changes in longitudinal emittance are observed.

\section{Discussion and conclusion}

\subsection{Comparison to SNS}

SNS is successfully operated superconducting proton linac. It has two $(\beta=0.61$ and $\beta=0.81$ ) sections of elliptical 6-cell $805 \mathrm{MHz}$ cavities. We benchmark our simulation of HOM power loss in $650 \mathrm{MHz}$ sections of Project-X linac against SNS. Figure 31 shows comparison of power loss due to resonance excitation of monopole HOM for Project-X HE $650 \mathrm{MHz}$ and SNS $\beta=0.81805 \mathrm{MHz}$ cavities. One can see, that expected HOM power 


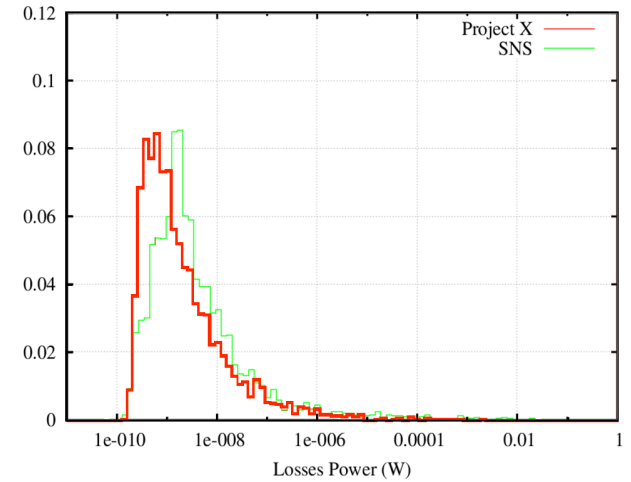

a)

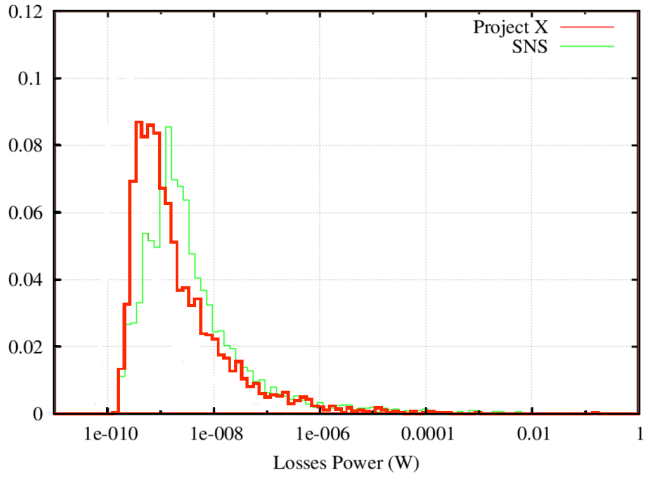

b)

Figure 31: Distribution of power loss per cavity for Project-X HE $650 \mathrm{MHz}$ and SNS $\beta=0.81805 \mathrm{MHz}$ cavities. (a) $Q_{L}=10^{8}$, (b) $Q_{L}=10^{9}$.

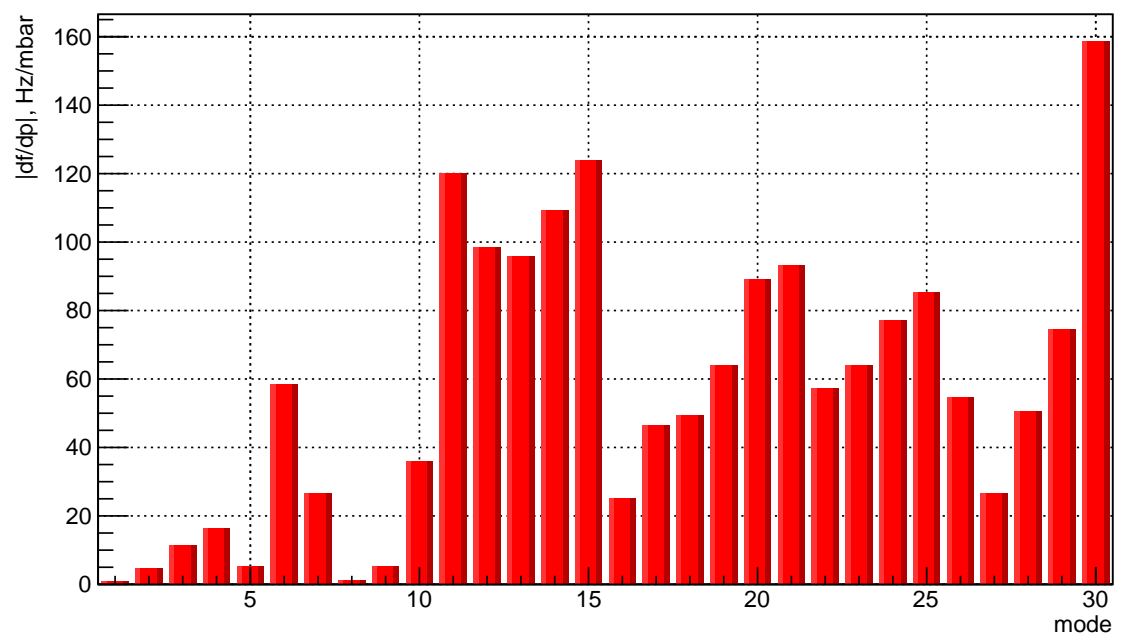

Figure 32: Microphonics for the monopole HOM in HE $650 \mathrm{MHz}$ cavity. 
loss in Project-X is smaller than in SNS linac, which is already quite small. Since SNS operates in pulsed regime, Lorentz force detuning of cavities during pulses and pulse-to-pulse jitter reduce HOM power loss even more. In Project-X linac, which operates in CW regime, similar effect of reduction of HOM losses may be expected from microphonics. Figure 32 shows sensitivity of the monopole HOMs frequencies to the variations of pressure in liquid Helium bath for HE $650 \mathrm{MHz}$ cavity. Cavity and Helium vessel are optimized in order to minimize effect of microphonics for operating mode. High order modes have larger detuning due to pressure variations, which is increasing with the mode frequency. Relatively large frequency variations of HOMs due to microphonics reduce probability of resonance excitation and detune HOMs from resonance if resonance conditions happen.

\subsection{Conclusion}

We study effects of high order modes in Project-X superconducting CW linac. The main goal of this study is to answer the question of whether HOM couplers/dampers are needed or not in SRF accelerating cavities of Project$\mathrm{X}$. Multiple issues related to the excitation of HOMs and their adverse effect on cryogenic losses and beam dynamics are addressed.

We find that beam breakup and "klystron"-type instabilities are negligible in $650 \mathrm{MHz}$ section of the linac. Incoherent HOM losses are small. Resonance excitation of dipole modes does not look to be an issue. Accidental resonance excitation of the monopole modes in $\mathrm{HE} 650 \mathrm{MHz}$ section may lead to the longitudinal emittance dilution, but the probability is small and HOM frequency can be moved from the dangerous resonance condition by detuning cavity operating mode and then tuning it back.

Based on this study we conclude that HOM couplers and dampers are not needed in Project-X linac.

\section{References}

[1] http://projectx.fnal.gov/

[2] H. Padamsee, J. Knobloch and T. Hays, RF Superconductivity for Accelerators, Wiley, New York, 1998.

[3] F. Marhauser et al., Design, fabrication and testing of medium-beta 650 MHz cavity prototypes for Project-X, IPAC 2011, San Sebastian, Spain, September 4-9, 2011. 
[4] V. Yakovlev et al., Concept EM design of the $650 \mathrm{MHz}$ cavities for the Project X, PAC 2011, New York, NY, USA, March 28-April 1, 2011.

[5] S.-H. Kim, SNS SCL Experiences, Project-X Collaboration Meeting, ORNL, Tennessee, USA, April 12-14, 2011.

[6] J. Sekutowicz, HOM couplers at DESY, HOM Workshop, Cornell University, Ithaca, New York, USA, October 11-13, 2010.

[7] D. G. Myakishev, V. P. Yakovlev, The New Possibilities of SuperLANS Code for Evaluation of Axisymmetric Cavities, PAC 1995, Dallas, Texas, USA, May 1-5, 1995.

[8] ILC Reference Design Report, http://www.linearcollider.org/about/ Publications/Reference-Design-Report

[9] http://xfel.desy.de/

[10] J. N. Corlett, et al., A Next Generation Light Source Facility at LBNL, PAC 2011, New York, NY, USA, March 28-April 1, 2011.

[11] A. Lunin, V. Yakovlev, S. Kazakov, Cavity loss factors of non-relativistic beams for Project X, PAC 2011, New York, NY, USA, March 28-April 1, 2011, TUP075.

[12] http://www.cst.com/

[13] M. H. Awida, P. Berrutti, I. V. Gonin, T. N. Khabiboulline, V. P. Yakovlev, SSR1 HOM Analysis and Measurements, IPAC 2012, New Orleans, Louisiana, USA, May 20-25, 2012.

[14] J. C. Slater, Microwave Electronics, D. Van Nostrand, Princeton, 1950.

[15] N. Solyak, J-P. Carneiro, V. Lebedev, S. Nagaitsev, N. Perunov, J-F. Ostiguy, A. Vostrikov, V. Yakovlev, Design of the Project X CW Linac, LINAC 2010, Tsukuba, Japan, September 12-17, 2010.

[16] J. Sekutowicz, HOM damping, ILC Workshop, KEK, Japan, November 13-15, 2004. 
[17] R. Sundelin, Frequency Spreads Caused By Manufacturing Tolerances, Cornell CLNS internal note SRF-830102-EXA, 1983.

[18] ANSYS HFSS, http://www.ansys.com/hfss

[19] A. Lunin, A. Saini, A. Sukhanov, N. Solyak, V. Yakovlev, Alternative Cavity for the HE Part of the Project X Linac, IPAC 2012, New Orleans, Louisiana, USA, May 20-25, 2012.

[20] T. Khabiboulline, Experiments on HOM Spectrum Manipulation in a ILC $1.3 \mathrm{GHz}$ Cavity, HOM Workshop, Cornell University, Ithaca, New York, USA, October 11-13, 2010.

[21] J. Tuckmantel, Do we need HOM dampers on superconducting cavities in proton linacs?, HOM Workshop, CERN, Switzerland, June 25-26, 2009.

[22] M. Schuh, F. Gerigk, J. Tuckmantel, C.P. Welsch, Higher order mode analysis of the SPL cavities, IPAC 2010, Kyoto, Japan, May 23-28, 2010 .

[23] J.-F. Ostiguy, P. Berrutti, J.-P. Carneiro, V. A. Lebedev, S. Nagaitsev, A. Saini, B. G. Shteynas, N. Solyak, V.P. Yakovlev, Status of the ProjectX CW Linac Design, IPAC 2012, New Orleans, Louisiana, USA, May 20-25, 2012. 